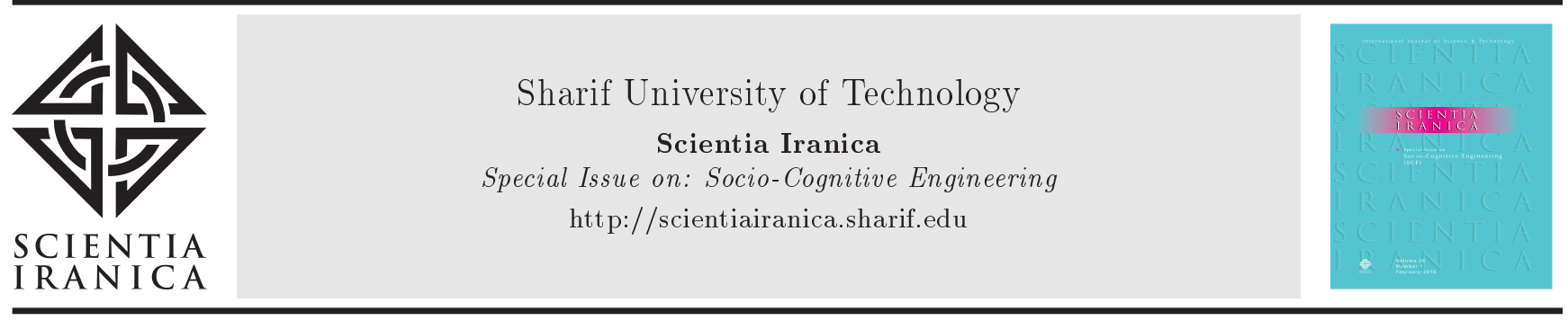

\title{
An efficient hardware implementation for a motor imagery brain computer interface system
}

\author{
A. Malekmohammadi, H. Mohammadzade*, A. Chamanzar, M. Shabany, and \\ B. Ghojogh
}

Department of Electrical Engineering, Sharif University of Technology, Tehran, Iran.

Received 9 August 2017; received in revised form 30 March 2018; accepted 4 August 2018

\author{
KEYWORDS \\ Brain Computer \\ Interface (BCI); \\ Electroencephalograph \\ (EEG); \\ Motor imagery; \\ Field Programmable \\ Gate Arrays (FPGA); \\ Separable Common \\ Spatio Spectral \\ Pattern (SCSSP); \\ Support Vector \\ Machine (SVM); \\ Linear Discriminant \\ Analysis (LDA).
}

\begin{abstract}
Brain Computer Interface (BCI) systems, which are based on motor imagery, enable humans to command artificial peripherals by merely thinking about the task. There is a tremendous interest in implementing BCIs on portable platforms, such as Field Programmable Gate Arrays (FPGAs) due to their low-cost, low-power and portability characteristics. This article presents the design and implementation of a Brain Computer Interface (BCI) system based on motor imagery on a Virtex-6 FPGA. In order to design an accurate algorithm, the proposed method avails statistical learning methods such as Mutual Information (MI), Linear Discriminant Analysis (LDA), and Support Vector Machine (SVM). It also uses Separable Common Spatio Spectral Pattern (SCSSP) method in order to extract features. Simulation results prove achieved performances of $73.54 \%$ for BCI competition III-dataset V, $67.2 \%$ for BCI competition IV-dataset 2a with all four classes, $80.55 \%$ for BCI competition IV-dataset 2 a with the first two classes, and $81.9 \%$ for captured signals. Moreover, the final reported hardware resources determine its efficiency as a result of using retiming and folding techniques from the VLSI architecture' perspective. The complete proposed BCI system achieves not only excellent recognition accuracy, but also remarkable implementation efficiency in terms of portability, power, time, and cost.

(C) 2019 Sharif University of Technology. All rights reserved.
\end{abstract}

\section{Introduction}

Nowadays, humans attempt to communicate with the outside world in a better and more convenient way. Although, at first glance, physical transactions seem essential for this goal, science is trying to make this relationship independent of physical actions.

Brain Computer Interface (BCI) is a system, enabling humans to control objects by merely mak-

\footnotetext{
*. Corresponding author.

E-mail addresses: malekmuhammadi@ee.sharif.edu (A.

Malekmohammadi); hoda@sharif.edu (H. Mohammadzade);

chamanzar_a@ee.sharif.edu (A.Chamanzar);

mahdi@sharif.edu (M. Shabany);

ghojogh_benyamin@ee.sharif.edu (B. Ghojogh)
}

ing decisions and thinking about them, i.e., without any sort of intervention of physical movement. In other words, a BCI system is an interface between the brain and computer to translate, process, and classify the electroencephalograph (EEG) signals [1]. After classification, this system sends commands to external devices, such as a wheelchair, to control it (Figure 1). A motor imagery system controls artificial devices by solely thinking about them. Movements of an artificial hand by thinking about its movement is one of the various examples of the applications of such systems, which is a predominant strategy for motor rehabilitation in stroke patients. Note that BCI systems are meant to be wearable, yet not sensible, in future applications; hence, it will be convenient and transparent to others. Moreover, BCI technology as a classification system is well established and has 


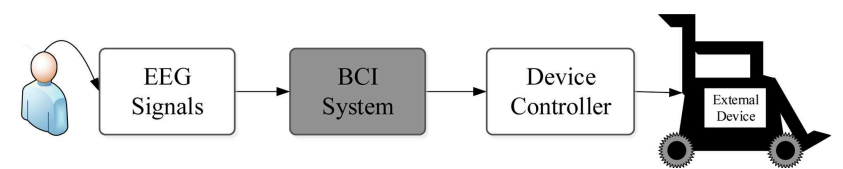

Figure 1. Functional model of a BCI system. The BCI system can command devices in order to help people with disabilities.

various applications, for instance, for patients with paraplegic devices. Therefore, any effort to improve its performance and/or efficient implementation is needed and valuable.

Although there exist several other controlling approaches, especially for patient or elderly care, motor imagery BCI system has some advantages over them. For instance, eye tracking [2] is not applicable in case the patients have visual or eye muscle problems, such as Amyotrophic Lateral Sclerosis (ALS) and Locked-In Syndrome (LIS), and, therefore, cannot control their eye muscles to move their eye in a certain direction [35]. Other problems of eye tracking can be found in dim light conditions or where the patient needs to notice his/her surrounding which would be dangerous for them to move their eye in other directions (e.g., when a patient in a wheelchair is passing on the street). Moreover, people with glasses can use BCI system much more conveniently than those with eye tracker can. Apart from their differences, both eye tracking and motor imagery $\mathrm{BCI}$ require a wearable device; eye tracker requires a pair of glasses to hold a camera to track eye, and BCI system requires a hat with electrodes. Moreover, note that despite the advantages of BCI system over eye tracking, eye tracker has the advantage of higher accuracy in comparison to motor imagery BCI.

In particular, in motor imagery BCI system, when a subject imagines moving, a typical desynchronization of upper alpha and beta rhythms is observed in the sensorimotor cortex [6], followed by a re-synchronization. This pattern of activation can be easily detected by EEG and used to feed a BCI system for different purposes. In fact, imagination movement tasks evoke EEG signal changes in a way that these changes can be detected in temporal and spatial traces of EEG rhythmic components, at specific electrodes (channels) located on subjects' scalps. Therefore, we can use electrophysiological properties of motor imagery as temporal, spatial and frequency characteristics to detect the mental task. This paper focuses on BCI systems that are based on translating EEG signals recorded through motor imagery.

A BCI system consists of units of signal acquisition, preprocessing, feature extraction, feature selection/reduction and classification. Signal acquisition unit records the brain activates such as EEG signals, while the pre-processing unit increases the signal-to- noise ratio of the signals. On the other side, feature extraction unit prepares features that are meaningful to the classification stage and omits outlier and artifact features. Then, the feature selection/reduction unit reduces the number of features and/or acquisition channels to decrease the dimension of feature vectors. Finally, classification unit classifies the features into logical control signals.

Solving a BCI problem includes two phases, i.e., the train and test phases. In the train phase, the parameters of the system are adjusted through learning from previously recorded samples. In the test phase, the new data are recorded and its class is estimated using the parameters adjusted in the train stage. Unfortunately, such algorithms require high-speed computers to process the steps of BCI, limiting their use and portability. Therefore, an efficient algorithm to meet these specifications is needed to be designed. In order to overcome this shortcoming, using hardware platforms such as Field Programmable Gate Arrays (FPGAs), which are more portable, less expensive, and power efficient, seems reasonable. Moreover, there are other reasons to use FPGA for implementing a BCI system. First, the parallelism of FPGAs can be used for high computational throughput, especially at low clock rates. Second, FPGAs are more flexible and more power-efficient than processors are. Third, FPGAs are more suitable for real-time embedded solutions [7].

This paper proposes an efficient high-accurate algorithm for classification of EEG signals for a BCI system. Moreover, the paper presents an efficient hardware implementation of the proposed $\mathrm{BCI}$ on a FPGA platform. The proposed algorithm is based on statistical learning methods, and the hardware implementation considers the optimization of power, area, and frequency to meet the targeted specifications of end applications. The achieved results show that the proposed BCI can classify EEG signals from BCI competition IV (4 classes), BCI competition III (3 classes), and our in-house signals (2 classes) with accuracy rates of $67.2 \%, 73.54 \%$, and $81.9 \%$, respectively. The hardware implementation results validate the successful implementation of the proposed method on hardware at the clock frequency of $560 \mathrm{kHz}$.

The remainder of this paper is organized as follows: In Section 2, the current literature is presented and discussed; in Section 3, all datasets used in this paper are presented; Section 4 describes the proposed methodology for the BCI system. Section 5 shows the simulation results of software, and Section 6 discusses signal processing concerns for hardware implementation. The detailed hardware implementation is presented in Section 7. Section 8 discusses some trade-offs between accuracy and hardware resources. In Section 9, the obtained hardware experimental results are reported. Finally, Section 10 concludes the paper. 


\section{Previous work}

There is a rich literature addressing the research in BCI systems, where each paper may consider a specific part of the system. The related literature can be divided into two main categories: algorithms and hardware implementation. In the following, state-of-the-art work from each category is briefly described.

\subsection{Previous work on algorithms}

There exists a large amount of literature on BCI algorithms and methods. Two main sub-categories are methods of feature extraction and those of classification:

1. Feature Extraction: Adaptive Auto-Regressive (AAR) is one of the suitable algorithms to extract features from EEG signals [8-10]. A classical approach to estimating time-varying AR-parameter is the segmentation-based approach. In this case, the data are divided into short segments and the AR parameters are estimated from each segment. The shorter the segment length, the higher the time resolution; however, it has the disadvantage of an increasing error in AR estimations. The AAR model appropriately describes the random behavior of EEG signals and provides parameters with a high time resolution. Moreover, by using AAR model, it is not necessary to use frequency band [11]. In [12], an AR model is utilized because of achieving better estimations of short time series compared to Fast Fourier Transform (FFT). AAR can also be used to remove artifacts, such as eye blinking and muscle movements, from EEG data, based on blind source separation [13].

However, the AR model cannot capture the transient features in EEG data, and the timefrequency information is not easily seen in the AR parameters [14]. Therefore, several researchers have used wavelet coefficients that provide localization of signal components with spectro-temporal characteristics [15-19]. The main benefit of wavelets is the time-frequency localization. The advantage of timefrequency localization is that the wavelet analysis changes the time-frequency aspect ratio, providing a good frequency localization at low frequencies for long time windows and good time localization at high frequencies for short time windows [20]. On the other hand, the Power Spectral Density (PSD) is another approach that indicates the distribution of power of a signal between different frequencies [21].

Various other algorithms have also been proposed that extract features in spectral or spatial domains such as the Common Spatial Patterns (CSP), classifying brain tasks. In fact, CSP, using the variance as a new feature, tries to extract features that are able to maximize the variance of a particular task and minimize the variance of the other [22]. A significant drawback of CSP is that it does not consider the spectral characteristics of the EEG signal [23]. To overcome this problem, many researchers have proposed several variants of CSP [24-27]. One of the promising algorithms for the feature extraction, which is an advanced version of CSP, is called Filter Bank Common Spatial Patterns (FBCSP) whose advantage is that it considers the spectral characteristics of the EEG signals [28,29]. Moreover, an advanced version of FBCSP is called Separable Common Spatio Spectral Pattern (SCSSP) [30], which overcomes all shortcomings of FBCSP, i.e., the high computational cost of training, lack of analysis of both spatial and spectral characteristics of signals, and lack of having a metric to rank the discriminatory power of extracted spatio-spectral features. In addition, CSP methods suffer from sensitivity to noise and, also, overfitting. In order to overcome these problems, different methods are proposed in the literature for regularizing CSP, namely Regularized CSP (RCSP). In [31], various RCSP algorithms are reviewed and summarized, as well as the standard CSP. Thereafter, four novel RCSP methods are proposed and evaluated which outperform previous RCSP methods.

2. Classification: Linear classifiers, such as Linear Discriminant Analysis (LDA) and Linear Support Vector Machine (SVM), can be applied to distinguish classes using linear functions [32]. Methods such as [33-36] have used these classifiers in BCI systems. LDA is suitable for online BCI systems because of a low computational cost, which persuades researchers to use it in motor imagery-based BCI systems. However, the main shortcoming of LDA is its linearity that can provide poor results on complicated nonlinear EEG data [37]. LDA can also be used for feature reduction in EEG signals [38], as is used in the proposed method, too. On the other hand, Linear-SVM uses a discriminant hyperplane to classify EEG features. This method is very popular because of good generalization properties [39], simplicity in implementation, and robustness [40]. Nonlinear classification, such as Bayesian and K-Nearest Neighbors (KNN), are used in [41-43]. Nonlinear methods show better classification performance than the linear methods; however, they are more complicated, especially from the implementation point of view. In spite of all these efforts, designing an efficient algorithm for classification in BCI systems, while considering the hardware constraints, is still a major challenge. 


\subsection{Previous work on hardware}

Most of the literature in which a full BCI system is implemented are concentrated on the steady-state visual Evoked Potential, which is an EEG signal response to the flickering visual stimulus [44], [45], [46]. However, in [47], a BCI system is implemented based on motor imagery, which includes Finite Impulse Response filter as a preprocessing, CSP as a feature extraction, and Mahalanobis distance as a classification, on Stratix IV FPGA Board with operational frequency of 200 $\mathrm{MHz}$. This paper proposes a more efficient hardware implementation with less hardware resources compared to the design in [47]. A more complete work of [47] is reported in [48]. A novel approach is introduced in [48], defining the tasks and working with devices by a state machine. The user can do the task or switch to other tasks by thinking about right and left hand movements.

There exist several other works on BCI systems with a hardware implementation perspective. For example, Wearable Mobile EEG-based Brain Computer Interface System (WMEBCIS) [49] is proposed for detecting drowsiness. A low-cost FPGA-based BCI multimedia control system is also proposed in [50]. Their proposed framework is used to control multimedia devices. In their work, they use Steady-State Visual Evoked Potential (SSVEP), which is light-emitting diode stimulation panel (several command symbols). SSVEP is also utilized in [51] to control environmental devices such as television. Controlling hospital bed nursing system in a FPGA-based BCI system is also addressed in [52].

\subsection{Best-performing methods on BCI competition datasets}

There are reported accuracies on BCI competitions available in BCI competition web site [53]. The best reported method (in [54]) on BCI competition IIIdataset $\mathrm{V}$, which has classified all three classes of this dataset, has used LDA to extract features from PSD values of the EEG signals and distance-based discriminator for classification. The best performing method (as reported in [55]) on BCI competition IVdataset 2a, which has classified all four classes of this dataset, has employed FBCSP and Naive Bayes Parzen Window as feature extraction and classification, respectively. However, the main shortcoming of these methods is that they provide low accuracies and are not suitable for hardware implementation as they have high computational complexity.

However, the methods proposed in [31] and [48], mentioned previously, outperform the best methods reported in [54] and [55], and can be considered as two of the best performing methods on BCI competition IV-dataset 2a. Nonetheless, it should be noticed that these two methods only classify the first two classes (movements of right and left hands).

\section{Description of datasets}

There are two available methods for recording the EEG signals, i.e., invasive and non-invasive methods. The former implants the electrodes in the brain through an operation; thus, it can be dangerous and harmful to the brain. However, the latter records the signals by electrodes on the head skin. In order to standardize the placement of the electrodes on the head, the 1020 standard has been introduced. In this standard, the distance of adjacent electrodes from each other is $10 \%$ or $20 \%$ of the whole distance between back (inion) and front (nasion) of the skull. This standard has improved over the years and has multiple versions. Figure 2 depicts the locations of almost all sensors in these standards.

Recording brain signals in the non-invasive approach is commonly called Electroencephalograph (EEG) [56]. The EEG method has some advantages and disadvantages. In fact, a lower price, high time resolution, and robustness of movement could be mentioned as its advantages, and low SNR, distilled water requirement and low spatial resolution are its disadvantages.

Capturing EEG signals, in the lab, consists of multiple steps. After the appearance of the fixation cross, a cue is performed to warn the experienced person to start thinking about a particular task, which is the motor imagery step. The last step is resting, which happens before starting the next experiment. Figure 3 illustrates the steps of the EEG signal capturing.

In this paper, three datasets are used to verify the proposed algorithm, which are detailed in the sequel.

\subsection{BCI competition III - dataset V}

One of the datasets used to evaluate the proposed algorithm is dataset V of BCI competition III [53]. This dataset was created by IDIAP Research Institute. Recorded signals are brain signals from three different persons, each of which is involved in the experiment for four times. All these four experiments were performed in one day with 5-10 minutes break between every session. The sampling rate is $512 \mathrm{~Hz}$ and 32 channels have been used to record the signals. There are both raw signals and power spectral densities available in this dataset; however, only raw signals are used in this paper. In this dataset, each person is asked to think about the following three tasks in different time slots:

- Imagination of repetitive self-paced left-hand movements;

- Imagination of repetitive self-paced right-hand movements;

- Generation of words beginning with the same random letter. 


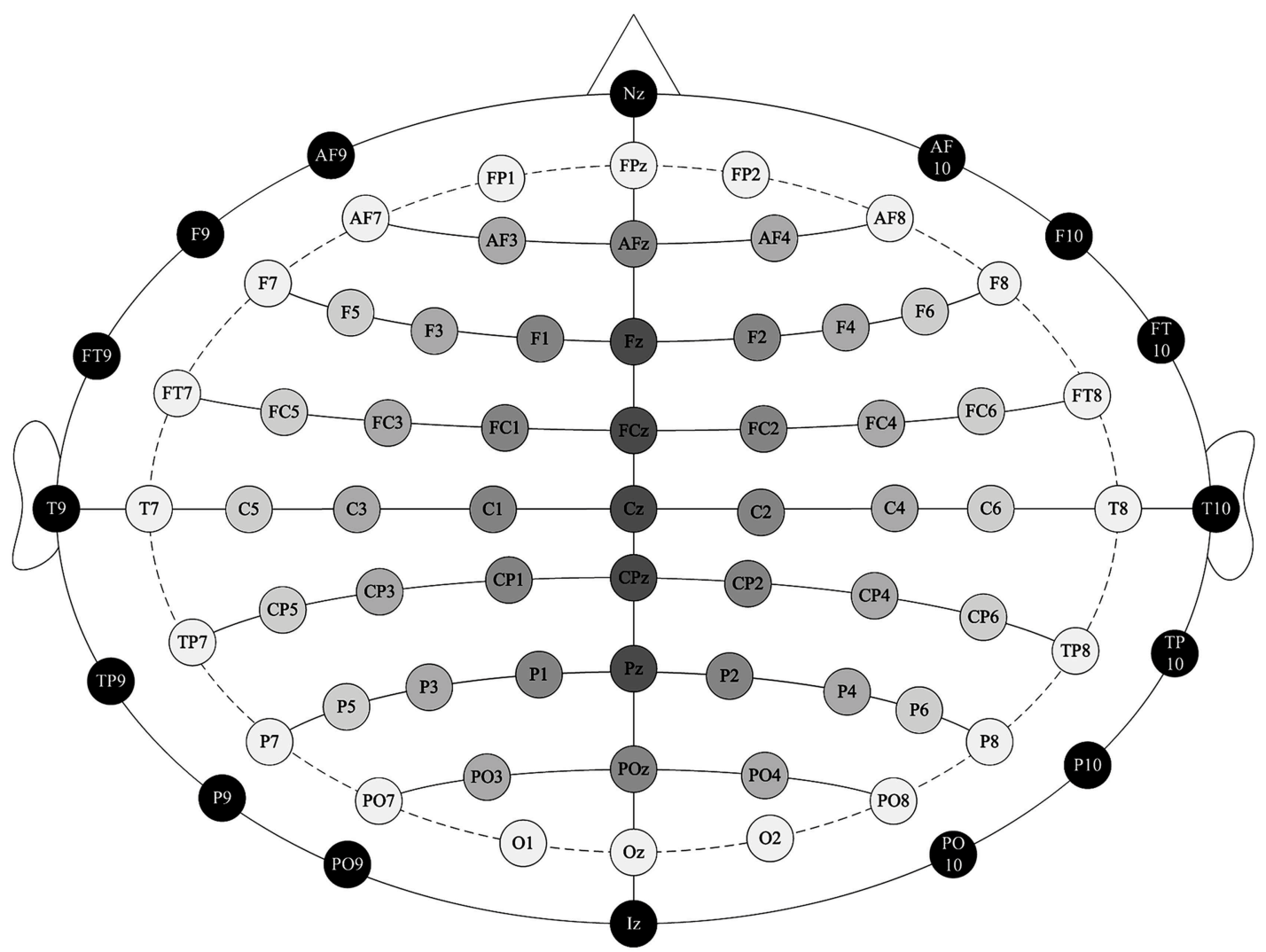

Figure 2. Locations of sensors in different versions of 10-20 standard.

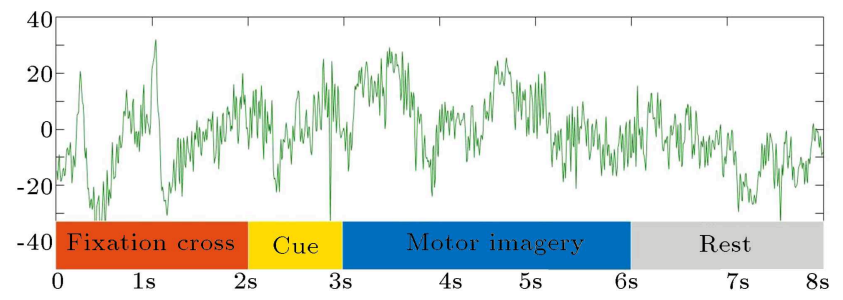

Figure 3. Timing scheme of the signal capturing paradigm. The paradigm includes fixation cross and cue followed by motor imagery and rest.

\subsection{BCI competition IV - dataset $2 a$}

The second dataset used in this paper is dataset $2 \mathrm{a}$ of BCI competition IV [53], consisting of signals from nine people. Signals from each person are captured in two sessions on different days. Each session consists of 6 parts and each part includes 48 experiments. In each experiment, every person is asked to do one of the following four motor imagery tasks:

- Imagination of left-hand movements;

- Imagination of right-hand movements;

- Imagination of both legs movements;

- Imagination of tongue movements.

The sampling rate is $50 \mathrm{~Hz}$ and data are filtered by a band-pass Butterworth filter.

\subsection{Captured signals}

In order to devise a local platform to evaluate the algorithms, we managed to record EEG signals by an Emotiv ${ }^{\circledR}$ system, including 14 channels with the sampling rate of $128 \mathrm{~Hz}$. The EEG electrodes have been placed in locations AF3, F7, F3, FC5, T7, P7, O1, O2, P8, T8, FC6, F4, F8, and AF4 based on International 10-20 system (see Figure 2). Eight persons were asked to imagine performing one of the following two tasks in each experiment:

- Imagination of left-hand movements;

- Imagination of right-hand movements.

Four experimental sessions were held in one day, each of them including 30 experiments. All subjects were sitting on a comfortable armchair in front of a computer screen during the recording session. A session takes eight seconds, including two seconds to show a fixation cross on the black screen at the beginning of session, one second for a cue pointing either to the left or right on the display, three seconds for performing the corresponding task in front of the black screen to avoid visual feedback, and two seconds in order to relax their minds (see Figure 3). The first three experimental sessions are used as training data, and the last one is used as test data. Before starting to record, all subjects were asked to practice with the experimental conditions 


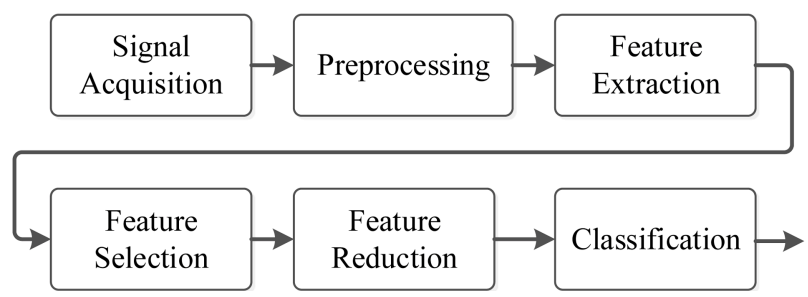

Figure 4. The overall block diagram of the proposed method. The proposed method consists of signal acquisition, preprocessing, feature extraction, feature selection, feature reduction, and finally classification.

for five minutes in order to reduce their stress and increase their concentration by becoming familiar with conditions. Subjects were asked not to move or blink during these three seconds of motor tasks in order to reduce the effects of artifacts such as electromyogram (EMG) and electrooculography (EOG). Signals were filtered by a highpass filter with $0.1 \mathrm{~Hz}$ cut-off frequency and, then, filtered by a notch filter with the frequency of $50 \mathrm{~Hz}$ to remove the power line effect. Moreover, note that, in the captured signals, subject 7 is about 60 years old, and the other subjects are young, around 23. The significant difference between the accuracy rates for subject 7 compared to the other subjects might be due to the older age of this subject.

\section{Methodology}

The method in this paper consists of several computational blocks, utilized in both train and test phases. Figure 4 shows different parts of this method. Each part will be discussed in detail in the following.

\subsection{Pre-processing}

Preprocessing, which tries to remove noises and artifacts, is crucial to obtaining high classification accuracy in BCI systems. In the proposed method, two techniques, i.e., DC-block and Laplacian filter, are used for this purpose.

1. DC-block: Filtering is an important step to remove unnecessary information from raw signals. In this paper, the fixed point DC blocker [57] is applied to remove the $\mathrm{DC}$ shifts. The $\mathrm{DC}$ component in EEG signals varies between different recording runs, even for a specific subject. This component does not include any information regarding the motor imagery task and may degrade the accuracy of the algorithm. Based on this fact and for the sake of achieving stability during all recording runs, the DC component should be omitted by means of a DC filter. An effective solution from the hardware implementation point of view is the DC-blocker, which performs the DC filtering by means of minimum hardware resources based on the following equation [57]:

$$
H(z)=\frac{1-z^{-1}}{1-\rho z^{-1}}
$$

where $\rho=0.996$.

2. Laplacian Filter: One of the most important limitations of EEG signals is their poor spatial resolution. One common technique in order to alleviate this problem is the Surface Laplacian (SL) filtering [58]. It is also used to remove the noises and artifacts whose origin may be outside of the skull [59], which eventually improves the Signal-toNoise Ratio (SNR). There are four different spatial filters, namely standard ear reference, Common Average Reference (CAR), small Laplacian and the large Laplacian. We employed the CAR method as it has been proven that the CAR is suitable for a communication system related to $\alpha$ and $\beta$ rhythms, which are the main frequency bands (8-12 $\mathrm{Hz}$ and 12-32 Hz, respectively) (some works, such as $[60,61]$, cite the range of $\beta$ rhythm as $18-30 \mathrm{~Hz}$ ) for motor imagery EEG signals [62]. In fact, in the CAR method, the potential of each channel (electrode) is subtracted by a weighted average of the next-nearest neighbor channels, according to their distance. If the distances are equal, it can be easily formulated as follows:

$$
V_{i}^{\mathrm{CAR}}=V_{i}^{\mathrm{ER}}-\frac{1}{n} \sum_{j=1}^{n} V_{j}^{\mathrm{ER}},
$$

where $V_{i}^{\mathrm{ER}}$ is the potential between the $i$ th electrode and the reference, and $n$ is the number of electrodes in the montage.

\subsection{Feature extraction}

Feature extraction is the process of distinguishing pertinent signal characteristic (i.e., features related to the user's intent) from unnecessary contents. The most popular methods in this field are AAR, wavelet-based, PSD and SCSSP, described in the sequel.

1. AAR: Autoregressive (AR) method is a simple, yet efficient, method for describing probabilistic behavior of a time sequence. In this paper, AAR is used for the adaptive estimation of the AR parameters [9]. The mathematical expression of this method can be written as follows:

$$
y_{k}=\sum_{i=k-p}^{k-1} a_{i} y_{i}+x_{k},
$$

where $x_{k}$ is a Gaussian noise with zero mean and variance $\sigma_{x}^{2}$, parameter $p$ is the degree of AR model, and $a_{i}$ 's are AR coefficients. Moreover, $k$ is a positive number, denoting the number of samples and is related to the signal duration as $k=t \times f_{0}$, where $f_{0}$ is the sampling frequency.

2. Wavelet: This method is able to produce good 
frequency localization in the time window, which is, in particular, appropriate for signals with transient nature such as EEG. The Wavelet coefficients are obtained through decomposition of the signal into different frequency bands. This decomposition is performed in multiple stages by means of consecutive low-pass and high-pass signal filtering. In this paper, this method is applied to find the coefficients of the frequency bands of $\alpha$ and $\beta$ rhythms as features of EEG signals.

3. $P S D$ : In this method, the power of the signal between different frequencies is considered as features. Herein, the value of PSD is computed in each $2 \mathrm{~Hz}$ frequency band within the $8-30 \mathrm{~Hz}$ to cover $\alpha$ and $\beta$ rhythms [21].

4. SCSSP: Separable Common Spatio Spectral Pattern (SCSSP), proposed in [30], improves the Common Special Patterns (CSP) method [22]. This method selects proper features of the signals as illustrated in Figure 5. Let us consider an EEG experiment with $N_{c h}$ channels (electrodes) and $N_{i}$ samples. After passing these signals through a set of $N_{f}$ band-pass filters, $N_{i}$ matrices with the size of $N_{f} \times N_{c h}$ are obtained. Let $X$ denote a $N_{f} \times N_{c h}$ matrix, and then its spectral $\left(\Phi_{i}\right)$ and special $\left(\Psi_{i}\right)$ covariance matrices are computed as follows:

$$
\begin{gathered}
\Phi_{i}=\frac{1}{N_{c h} N_{i}} \sum_{n=1}^{N_{i}} X X^{T}, \\
\Psi_{i}=\frac{1}{N_{f} N_{i}} \sum_{n=1}^{N_{i}} X^{T} X,
\end{gathered}
$$

where $N_{i}$ is the number of training samples $X$. Then, by solving the generalized eigenvalue problems in Eq. (5):

$$
\begin{aligned}
& \Phi_{1} W_{L}=\left(\Phi_{1}+\Phi_{2}\right) W_{L} \Lambda_{L}, \\
& \Psi_{1} W_{R}=\left(\Psi_{1}+\Psi_{2}\right) W_{R} \Lambda_{R},
\end{aligned}
$$

desired eigenvectors $\left(W_{L}\right.$ and $\left.W_{R}\right)$ and eigenvalues $\left(\Lambda_{L}\right.$ and $\left.\Lambda_{R}\right)$ are calculated.
Following this calculation, components $\lambda^{k}$ are computed as follows:

$$
\lambda^{k}=\frac{\lambda_{L}^{i[k]} \lambda_{R}^{j[k]}}{\lambda_{L}^{i[k]} \lambda_{R}^{j[k]}+\left(1-\lambda_{L}^{i[k]}\right)\left(1-\lambda_{R}^{j[k]}\right)},
$$

where $\lambda_{L}^{i[k]}$ and $\lambda_{R}^{j[k]}$ are components of $\Lambda_{L}$ and $\Lambda_{R}$ eigenvalue matrices, respectively, and $k$ is between 1 and $N_{f} N_{c h} \lambda^{k}$ s values are then sorted in descending order, and the first $d$ is selected in order to determine the corresponding indices $i[k]$ and $j[k]$. Therefore, the features are obtained as follows:

$$
\begin{aligned}
y_{k}= & \mathbf{w}_{L, i[k]}^{T} X \mathbf{w}_{R, j[k]} \quad \text { for } \quad k \in \mathcal{R}, \\
\mathcal{R}= & \left\{1, N_{f} N_{c h}, 2,\left(N_{f} N_{c h}-1\right), \ldots,\right. \\
& \left.\frac{d}{2},\left(N_{f} N_{c h}-\frac{d}{2}+1\right)\right\}
\end{aligned}
$$

where $\mathbf{w}_{L, i[k]}$ and $\mathbf{w}_{R, j[k]}$ are the eigenvectors in $W_{L}$ and $W_{R}$ corresponding to $\lambda_{L}^{i[k]}$ and $\lambda_{R}^{j[k]}$, and $y_{k}$ can also be found as follows:

$$
y_{k}=\left(\mathbf{w}_{R, j[k]} \otimes \mathbf{w}_{L, i[k]}\right)^{T} X,
$$

where $\otimes$ denotes Kronecker product.

It is suggested to use the normalized log-power features instead, which are calculated as follows:

$$
z_{k}=\log \left(\frac{\operatorname{var}\left(y_{k}\right)}{\sum_{k \in \mathcal{R}} \operatorname{var}\left(y_{k}\right)}\right),
$$

where $z_{k}$ is the $k$ th feature. The feature vector is then constructed as $Z=\left[z_{1}, z_{N_{f} N_{c h}}, \ldots\right]^{T}$.

Notice that the SCSSP method is a binaryclassification algorithm. To generalize it for multi-class purposes, several auxiliary approaches, such as OneVersus-Rest (OVR), can be used.

Finally, the band-pass filtering is performed using Chebyshev type II filters of order 6 and bandwidth of $4 \mathrm{~Hz}$. A total of $N_{f}=4$ filters are used to cover $\alpha$ and

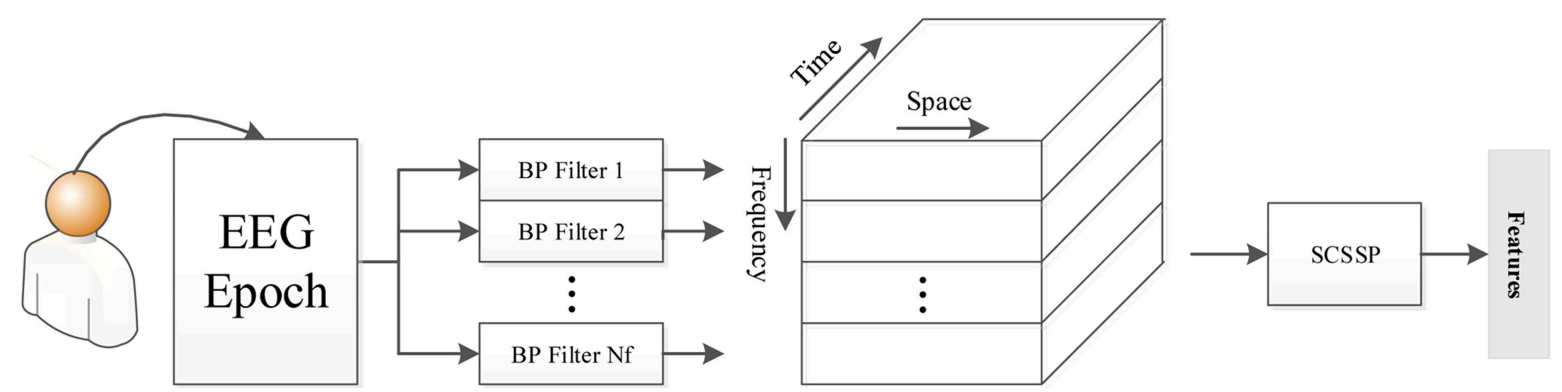

Figure 5. Structure of SCSSP algorithm. The EEG signals with $N_{i}$ samples obtained from $N_{c h}$ channels pass through $N_{f}$ band-pass filters, and a three-dimensional matrix with the size of $N_{f} \times N_{c h} \times N_{i}$ is obtained. Afterwards, the SCSSP algorithm extracts proper features out of it, as explained in the manuscript. 
$\beta$ rhythms. The reasons for using this type of filter are as follows: IIR filters need extremely fewer coefficients than FIR filters do; therefore, they require much less hardware resources to implement in comparison to FIR filters. Hence, an IIR filter type is applied. Among IIR filters, Elliptic and Chebyshev type I filters have ripples in their band pass, causing adverse influence on the final results. The Chebyshev type II Filter is sharper in the cut-off frequency than Butterworth Filter is. Therefore, better accuracy can be expected from Chebyshev type II filter.

\subsection{Normalization}

Normalization of features limits their dynamic range and improves the accuracy of the algorithm. It can be performed in both linear and non-linear forms. Assume that $m_{k}$ and $\sigma_{k}$ are mean and standard deviation of the $k$ th dimension of feature vector $(Z)$, respectively:

$$
\begin{aligned}
m_{k} & =\frac{1}{N} \sum_{i=1}^{N} Z_{i k}, \\
\sigma_{k}^{2} & =\frac{1}{N-1} \sum_{i=1}^{N}\left(Z_{i k}-m_{k}\right)^{2}, \quad k=1,2, \ldots, l,
\end{aligned}
$$

where $N$ is the number of training samples. Then, the linear normalization of the train and test $Z_{k}$ is calculated as follows:

$$
\hat{Z}_{k}=\frac{Z_{k}-m_{k}}{\sigma_{k}}
$$

\subsection{Feature election}

To reduce the number of obtained features efficiently, the following two methods can be used:

1. FDR: Fisher Discriminant Ratio (FDR) is a ratio that considers the best features by maximizing the distance between the means of the classes and minimizing the variance within each class. Features that satisfy longer FDR are better ones to be selected.

If $\mu_{i}$ and $\sigma_{i}$ denote the mean and standard deviation of feature $Z_{k}$ in the $i$ th class, respectively, the FDR for this feature is calculated as follows:

$$
\mathrm{FDR}=\sum_{i=1}^{C} \sum_{j=1}^{C} \frac{\left(\mu_{i}-\mu_{j}\right)^{2}}{\sigma_{i}^{2}+\sigma_{j}^{2}},
$$

where $C$ is the number of classes. A higher FDR value implies that the feature has a better contrast to classify classes.

2. $M I$ : The mutual information $I(Z, \omega)$ between variable $Z$ from the feature space and class labels $\omega=\left\{\omega_{1}, \omega_{2}, \ldots, \omega_{c}\right\}$ is defined as follows:

$$
I(Z ; \omega)=\sum_{Z} \sum_{i=1}^{c} p\left(Z, \omega_{i}\right) \log \left(\frac{p\left(Z, \omega_{i}\right)}{p(Z) p\left(\omega_{i}\right)}\right),
$$

where $p($.$) denotes the probability function. The$ more independent variables $Z$ and classes are, the less mutual information the feature will have. It should be noted that the output value of $I$ is always greater than zero. Features satisfying higher quantity of MI with regard to the classes are selected as better features [63-65].

\subsection{Feature reduction}

After selecting proper features, still many of them may have dummy information. To reduce the dimension of features, several methods have been proposed, two of which are described in the following:

1. LDA: Linear Discriminant Analysis (LDA), also known as Fisher LDA, is a popular method for dimension reduction and classification [66]. The important advantage of LDA is that it amends and improves the total achieved accuracy while providing a very simple methodology. As LDA requires very low computational complexity, it is a worthy selection for BCI systems [67].

LDA projects the data using a transformation matrix $W$ onto a new space with lower dimensions. If the number of classes is $C$, the new feature dimension $(d)$ will be $C-1$. LDA tries to minimize within-class scatter $S_{w}$ and maximize between-class scatter $S_{b}$ formulated as follows:

$$
\begin{aligned}
& S_{w}=\sum_{j=1}^{C} \sum_{i=1}^{n_{i}}\left(z_{i}^{j}-\mu_{j}\right)\left(z_{i}^{j}-\mu_{j}\right)^{T}, \\
& S_{b}=\sum_{j=1}^{C}\left(\mu_{j}-\mu\right)\left(\mu_{j}-\mu\right)^{T},
\end{aligned}
$$

where $z_{i}^{j}$ is the $i$ th sample of class $j, \mu_{j}$ is the mean of class $j, n_{i}$ is the number of samples in class $j$, and $\mu$ is the mean of means of all classes. The transformation matrix $W$ and, therefore, the new feature space are constructed by eigenvectors corresponding to the biggest eigenvalues $(d=C-1)$ of $S_{w}^{-1} S_{b}$ matrix. The new features are obtained by projection of features onto this new feature space.

2. PCA: Principle Component Analysis (PCA) is another popular method for dimension reduction which uses an orthogonal transformation, $Y=$ $W^{T} Z$, to convert data $Z$ into uncorrelated data $Y$. Matrix $W$ is constructed by eigenvectors of the covariance matrix of $Z$ data corresponding to the largest $d$ eigenvalues, containing most information of original space. By comparing LDA and PCA methods, one can easily see that LDA increases the resolution between classes, while PCA reduces the error of data compression. 


\subsection{Classification}

There are many methods available for classification. The following classification methods are tested in this work:

1. KNN: K-Nearest Neighbor is a simple classification method that finds $K$ nearest neighbors from the training data for each new point in testing data. The class with the largest number of samples among $K$ samples is the winner class for the test sample. The distance is calculated using these following measures: (I) Euclidean distance, (II) Absolute distance.

2. Bayes: Supposing that the data is $D$-dimensional as $x=\left[x_{1}, \ldots, x_{D}\right]^{T}$, the probability distribution of dimension $x_{d}$ over all the training samples of class $j$ is as follows:

$$
\begin{aligned}
& p\left(x_{d} \mid \text { class }: j\right)=\frac{1}{\sqrt{2 \pi \sigma_{d, j}^{2}}} e^{-\frac{\left(x_{i}-\mu_{d, j}\right)^{2}}{2 \sigma_{d, j}^{2}}} \\
& \propto \frac{1}{\sigma_{d, j}} e^{-\frac{\left(x_{i}-\mu_{d, j}\right)\left(x_{i}-\mu_{d, j}\right)}{\sigma_{d, j}^{2}}} \\
& \propto \frac{1}{\sigma_{d, j}}\left[\sinh \left(f\left(x_{i}\right)\right)+\cosh \left(f\left(x_{i}\right)\right)\right],
\end{aligned}
$$

where $\sigma_{d, j}$ and $\mu_{d, j}$ are respectively standard deviation and mean of the $d$ th attribute of all training samples in class $j$, and $f\left(x_{i}\right)=-\left(x_{i}-\mu_{d, j}\right)\left(x_{i}-\right.$ $\left.\mu_{d, j}\right) / \sigma_{d, j}^{2}$.

If there are $C$ classes indexed by $j=$ $\{1, \ldots, C\}$, the estimated class of the a test sample $x=\left[x_{1}, \ldots, x_{D}\right]^{T}$ is found by the following:

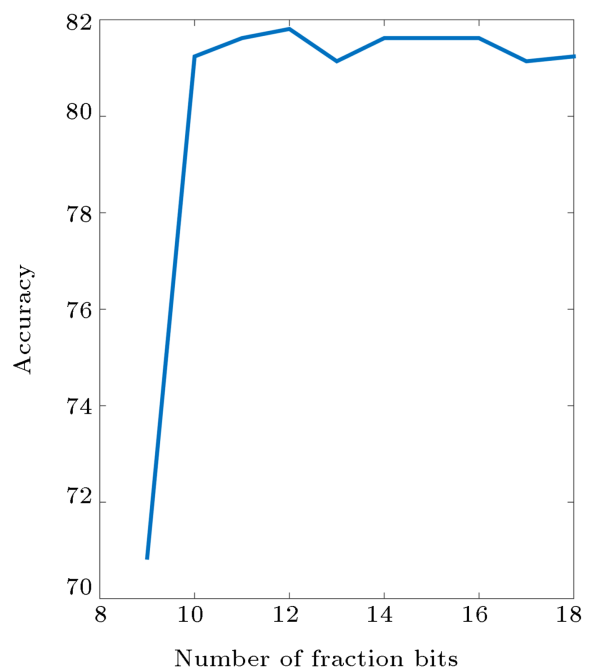

(a)

$$
\begin{aligned}
\hat{y}= & \arg \max _{j} p(\operatorname{class}: j) \prod_{d=1}^{D} p\left(x_{d} \mid \text { class }: j\right) \\
& \propto \arg \max _{j} \prod_{d=1}^{D} p\left(x_{d} \mid \text { class }: j\right)
\end{aligned}
$$

because when uniform probability distribution is assumed for classes, $p$ (class : $j$ ) can be discarded.

3. SVM: Support Vector Machine (SVM) is one of the well-known classification algorithms and has been widely used in BCI due to its simplicity. SVM constructs hyperplanes to separate the feature vectors into several classes. These hyperplanes maximize the margins, that is, the distance between the nearest training samples and the hyperplanes [68]. The goal during the training process is to find the separating hyperplane with the possible largest margin from the edge of classes [66].

\section{Performance results}

In this paper, for each part of the system, several methods were investigated and tested to find the optimum approach. Given the fact that the algorithm was going to be implemented on hardware, all values were considered fixed point in experiments performed in MATLAB. Twenty bits were found to be sufficient for each value of each channel, in which 11 bits were considered for the decimal part, 8 bits for the integer section, and one sign bit. The number of bits is carefully determined using the bit-loading process. This means that through extensive simulations, the dynamic ranges of the variables were monitored and logged. As shown in Figure 6(a), the number of bits associated to fractional part suffices to be 11 to have stable and good total accuracy in the experiments.

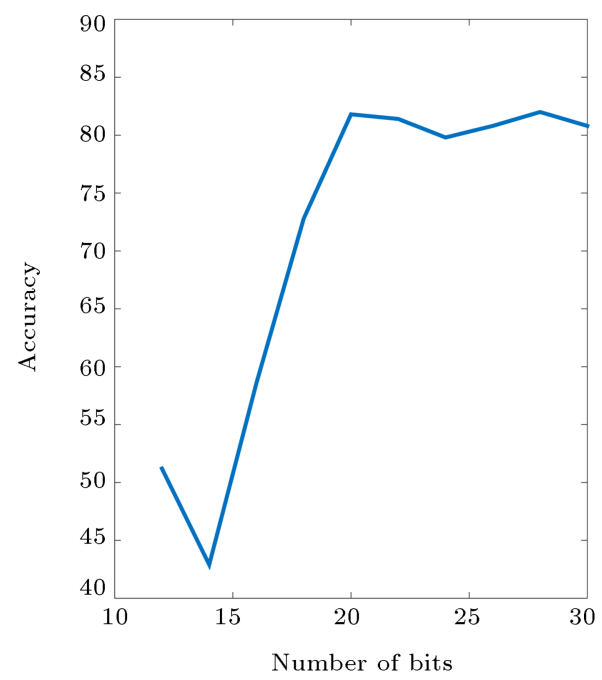

(b)

Figure 6. Effect of number of bits on the total accuracy: (a) Effect of number of bits associated to fractional part, and (b) effect of number of bits associated to the whole number. 


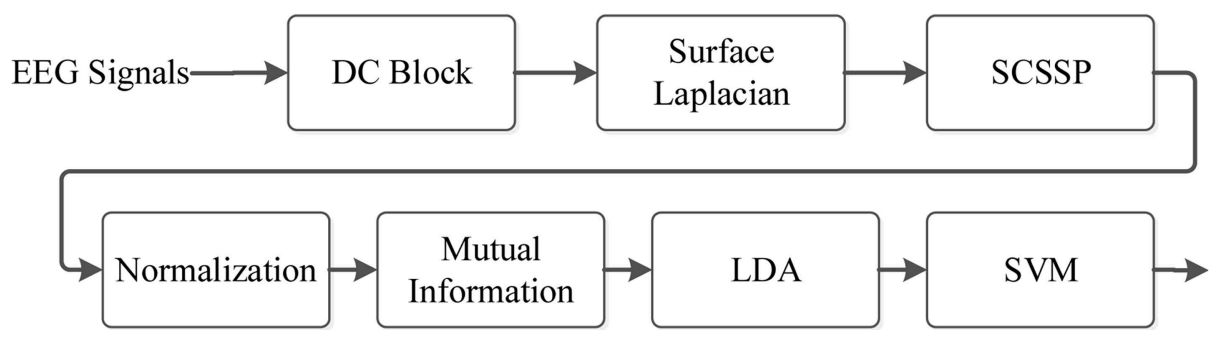

Figure 7. Final structure of the proposed method. DC block and surface Laplacian pre-process data. SCSSP, mutual information, LDA, and SVM are respectively selected to be used for feature extraction, selection, reduction, and classification.

Moreover, Figure 6(b) verifies that 20 bits are sufficient for representing the whole number in this work.

Among feature extraction methods, SCSSP performs better than others do, for all different classification methods (see Table 1). Therefore, SCSSP is the best choice for the feature extraction. As can be seen in this table, the normalization of features improves the accuracy rate by $5.4 \%$. MI and LDA methods perform the best among feature selection and reduction methods, respectively. The results show that Bayesian approach outperforms other classification methods with a slight improvement compared to the linear SVM. However, because of its low implementation cost on hardware, linear SVM is selected for classification in this work (in the next sections, the numeric comparisons of linear SVM and Bayesian classifiers are reported for both software and hardware implementations). In conclusion, the final structure of the proposed method is depicted in Figure 7.

Overall results of the algorithm (for complete number of classes) are listed in Table 1, showing better performance than the best reported results of BCI competitions [54,55] and also recent state-of-the-art papers $[31,48]$ for both datasets $(67.2 \%$ for BCI competition IV, dataset $2 \mathrm{a} ; 73.54 \%$ for BCI competition III, dataset V). Moreover, the results show a high performance of $81.9 \%$ for captured signals, reflecting the excellent performance of the proposed algorithm. Notice that, in the captured dataset, as mentioned in Section 3.3, subject 7 is much older than the other subjects are. This may explain the significant difference between the accuracy rates of this subject and the other subjects.

Several recent state-of-the-art papers working on motor imagery (such as $[31,48]$ ) have considered merely two classes of right- and left-hand movements for experiments. To compare the proposed method with similar state-of-the-art methods, we evaluate this work with two mentioned classes, too. The experiment is performed on BCI competition IV, dataset 2a. According to Table 1, this work outperforms [31] with a slight improvement; however, it does not reach the performance of [48]. One of the reasons for not outperforming [48] is that our proposed system tries to make a balance between accuracy and hardware efficiency by considering hardware issues, too, while accuracy is a matter of much concern in [48] resulting in lower hardware efficiency. The other reasons for the lower accuracy of our proposed method, compared to [48] and some details of [31] and [48], are explained in the following.

This work differs from [31] in some important items. First, in [31], several additional subjects are used as a prior knowledge (for regularization) in the training phase. Obviously, the use of additional subjects in training improves the performance. Additional subjects might not be always available. Secondly, they have not proposed the hardware implementation of their method; thus, their simulations are in the floating point while the reported results of this paper are in fixed-point. In fact, the software experiments of the proposed method in this paper are performed in fixed point in order to be realistic for hardware implementation. Third, [31] uses CSP and RCSP, while this work uses SCSSP which has significant advantages over CSP method. Fourth, they have used three pairs $\left(N_{f}=3\right.$ pairs) Butterworth filters with order 5 , while four $\left(N_{f}=4\right)$ Chebyshev filters are used in this work with order 6 .

In [48], a motor imagery embedded system is proposed in both hardware and software frameworks. Their method consists of adjusting filters in preprocessing, using the CSP method for feature extraction and utilizing Mahalanobis distance as a classifier. Several differences between [48] and this work are as follows. First, in [48], the filter is optimized for each subject according to intrinsic characteristics of the subject. However, identical filters are used for all subjects in this paper. For instance, for the best total accuracy in [48], the degrees of optimum FIR filters for subjects S2, S4, and S6 are respectively 221, 146, and 442. However, the orders of Chebyshev filters in this paper are all six, which are much easier to implement and consume much less area and power in hardware. Second, because of this optimization of every subject, [48] is subject-dependent in contrast to the proposed method in this paper. Being subjectdependent has serious disadvantages, such as the need 
Table 1. Algorithm experiments and results. The first five row blocks of table report the evaluations for obtaining optimum framework. The feature extraction methods are evaluated for four different classification methods. The experiments of feature extraction methods are done on BCI competition III, dataset V. The other evaluations are done on BCI competition IV, dataset 2a. The performances of overall framework on the three datasets are reported in the last row block of the table. All the experiments of this table are performed using MATLAB.

\begin{tabular}{|c|c|c|c|c|c|c|c|c|c|c|c|}
\hline & & S1 & $\mathrm{S} 2$ & S3 & $\mathrm{S} 4$ & S5 & S6 & $\mathrm{S} 7$ & S8 & S9 & Average \\
\hline \multicolumn{12}{|c|}{ Feature extractio } \\
\hline \multirow{4}{*}{ AAR } & $\mathrm{KNN}$ & $76.97 \%$ & $60.89 \%$ & $39.58 \%$ & $\times$ & $\times$ & $\times$ & $\times$ & $\times$ & $\times$ & $59.15 \%$ \\
\hline & Bayesian & $76.33 \%$ & $61.52 \%$ & $39.16 \%$ & $\times$ & $\times$ & $\times$ & $x$ & $\times$ & $\times$ & $59.00 \%$ \\
\hline & LDA & $66.74 \%$ & $60.25 \%$ & $47.79 \%$ & $\times$ & $\times$ & $\times$ & $\times$ & $\times$ & $\times$ & $58.26 \%$ \\
\hline & SVM & $77.19 \%$ & $61.95 \%$ & $42.32 \%$ & $\times$ & $x$ & $\times$ & $x$ & $\times$ & $\times$ & $60.48 \%$ \\
\hline \multirow{4}{*}{ Wavelet } & $\mathrm{KNN}$ & $73.35 \%$ & $56.45 \%$ & $47.16 \%$ & $\times$ & $\times$ & $\times$ & $x$ & $\times$ & $\times$ & $58.99 \%$ \\
\hline & Bayesian & $72.49 \%$ & $52.64 \%$ & $45.05 \%$ & $x$ & $x$ & $x$ & $x$ & $\times$ & $x$ & $56.72 \%$ \\
\hline & LDA & $68.66 \%$ & $57.93 \%$ & $48.00 \%$ & $x$ & $x$ & $x$ & $x$ & $\times$ & $\times$ & $58.2 \%$ \\
\hline & SVM & $71.43 \%$ & $54.76 \%$ & $43.79 \%$ & $x$ & $\times$ & $x$ & $\times$ & $\times$ & $\times$ & $56.66 \%$ \\
\hline \multirow{4}{*}{ PSD } & $\mathrm{KNN}$ & $71.75 \%$ & $52.16 \%$ & $41.84 \%$ & $\times$ & $\times$ & $\times$ & $\times$ & $x$ & $x$ & $55.25 \%$ \\
\hline & Bayesian & $64.80 \%$ & $53.12 \%$ & $45.00 \%$ & $\times$ & $\times$ & $\times$ & $\times$ & $\times$ & $\times$ & $54.31 \%$ \\
\hline & LDA & $72.42 \%$ & $54.12 \%$ & $45.20 \%$ & $\times$ & $x$ & $x$ & $x$ & $\times$ & $\times$ & $57.24 \%$ \\
\hline & SVM & $70.64 \%$ & $52.64 \%$ & $46.44 \%$ & $\times$ & $\times$ & $\times$ & $x$ & $\times$ & $\times$ & $56.57 \%$ \\
\hline \multirow{4}{*}{ SCSSP } & KNN & $81.02 \%$ & $55.18 \%$ & $54.53 \%$ & $\times$ & $\times$ & $\times$ & $x$ & $\times$ & $\times$ & $63.58 \%$ \\
\hline & Bayesian & $82.52 \%$ & $58.35 \%$ & $53.26 \%$ & $\times$ & $\times$ & $x$ & $\times$ & $\times$ & $\times$ & $64.71 \%$ \\
\hline & LDA & $79.53 \%$ & $61.31 \%$ & $55.79 \%$ & $x$ & $x$ & $x$ & $x$ & $\times$ & $\times$ & $65.54 \%$ \\
\hline & SVM & $79.1 \%$ & $59.20 \%$ & $53.47 \%$ & $\times$ & $\times$ & $x$ & $x$ & $\times$ & $x$ & $63.92 \%$ \\
\hline \multicolumn{12}{|c|}{ Normalization } \\
\hline Norm & & $79.9 \%$ & $56.3 \%$ & $80.2 \%$ & $63.9 \%$ & $40.3 \%$ & $44.8 \%$ & $77.4 \%$ & $78.1 \%$ & $77.1 \%$ & $66.4 \%$ \\
\hline Not-norm & & $77.1 \%$ & $56.3 \%$ & $73.6 \%$ & $60.4 \%$ & $33.0 \%$ & $37.2 \%$ & $69.8 \%$ & $72.2 \%$ & $69.4 \%$ & $61.0 \%$ \\
\hline \multicolumn{12}{|c|}{ Feature selection } \\
\hline FDR & & $77.7 \%$ & $58.3 \%$ & $84.7 \%$ & $63.5 \%$ & $37.1 \%$ & $40.7 \%$ & $81.9 \%$ & $71.1 \%$ & $75.6 \%$ & $65.6 \%$ \\
\hline MI & & $77.7 \%$ & $58.6 \%$ & $87.1 \%$ & $62.8 \%$ & $41.6 \%$ & $43.7 \%$ & $81.9 \%$ & $74.3 \%$ & $70.4 \%$ & $66.5 \%$ \\
\hline \multicolumn{12}{|c|}{ Feature reduction } \\
\hline LDA & & $79.8 \%$ & $56.2 \%$ & $80.2 \%$ & $63.8 \%$ & $40.2 \%$ & $44.7 \%$ & $77.4 \%$ & $78.1 \%$ & $77.0 \%$ & $66.4 \%$ \\
\hline $\mathrm{PCA}$ & & $72.9 \%$ & $53.1 \%$ & $80.2 \%$ & $61.1 \%$ & $37.8 \%$ & $40.6 \%$ & $71.8 \%$ & $70.1 \%$ & $67.7 \%$ & $61.7 \%$ \\
\hline \multicolumn{12}{|c|}{ Classification } \\
\hline KNN & & $78.1 \%$ & $57.2 \%$ & $83.3 \%$ & $67.7 \%$ & $39.5 \%$ & $36.8 \%$ & $75.0 \%$ & $78.8 \%$ & $74.6 \%$ & $65.7 \%$ \\
\hline Bayes & & $78.1 \%$ & $55.9 \%$ & $82.9 \%$ & $67.7 \%$ & $43.4 \%$ & $40.6 \%$ & $78.8 \%$ & $77.7 \%$ & $78.1 \%$ & $67.0 \%$ \\
\hline Linear SVM & & $79.8 \%$ & $56.2 \%$ & $80.2 \%$ & $63.8 \%$ & $40.2 \%$ & $44.7 \%$ & $77.4 \%$ & $78.1 \%$ & $77.0 \%$ & $66.4 \%$ \\
\hline \multicolumn{12}{|c|}{ Performance results } \\
\hline BCI competition III & Best of $[54]$ & $80 \%$ & $70 \%$ & $56 \%$ & $\times$ & $\times$ & $\times$ & $x$ & $\times$ & $\times$ & $68.7 \%$ \\
\hline (Dataset V, 3 classes) & This work & $86.6 \%$ & $78.0 \%$ & $56.0 \%$ & $\times$ & $\times$ & $\times$ & $x$ & $\times$ & $\times$ & $73.5 \%$ \\
\hline BCI competition IV & Best of $[55]$ & $68 \%$ & $42 \%$ & $75 \%$ & $48 \%$ & $40 \%$ & $27 \%$ & $77 \%$ & $75 \%$ & $61 \%$ & $57 \%$ \\
\hline (Dataset $2 \mathrm{a}, 4$ classes) & This work & $79.2 \%$ & $56.3 \%$ & $87.5 \%$ & $63.9 \%$ & $41.0 \%$ & $46.9 \%$ & $81.9 \%$ & $76.0 \%$ & $72.2 \%$ & $67.2 \%$ \\
\hline BCI competition IV & Best of $[31]$ & $88.89 \%$ & $54.86 \%$ & $96.53 \%$ & $70.14 \%$ & $65.97 \%$ & $61.81 \%$ & $81.25 \%$ & $95.83 \%$ & $90.97 \%$ & $79.4 \%^{1}$ \\
\hline (Dataset $2 \mathrm{a}, 2$ classes) & Best of $[48]$ & $97.36 \%$ & $89.47 \%$ & $89.47 \%$ & $92.1 \%$ & $97.36 \%$ & $92.1 \%$ & $84.22 \%$ & $94.73 \%$ & $92.1 \%$ & $92.10 \%^{2}$ \\
\hline & This work & $91.67 \%$ & $59.72 \%$ & $95.83 \%$ & $77.08 \%$ & $67.36 \%$ & $69.44 \%$ & $78.47 \%$ & $97.22 \%$ & $88.19 \%$ & $80.55 \%$ \\
\hline Captured signals & This work & $93.6 \%$ & $93.6 \%$ & $96.8 \%$ & $83.9 \%$ & $71.0 \%$ & $71.0 \%$ & $58.0 \%$ & $87.1 \%$ & $x$ & $81.9 \%$ \\
\hline
\end{tabular}

${ }^{1}$ In [31], several additional subjects are used for regularization in training phase.

In addition, in contrast to evaluations of this work, experiments in [31] are in floating point simulation.

${ }^{2}$ In [48], in contrast to this work, the filters are not identical for all subjects, and filter of each subject is optimized according to the subject's characteristics. Therefore, [48] is subject-dependent.

Moreover, the orders of filters are significantly high in comparison to orders of filters in this work.

In addition, as our best understanding from [48], the training and testing sets differ from the standard sets in the dataset.

to train the whole system again by introducing new subjects, having slower training phase, difficulty in the expansion and mass production, etc. Third, in [48], the ratio of training to test samples is $60 \% / 40 \%$. However, the ratio of the training to test in the standard BCI competition IV, dataset $2 \mathrm{a}$, is $50 \% / 50 \%$, which is used in our work for evaluation. In addition, as is reported in the following sections, this work in this paper highly outperforms [48] in the total consumed power.

Figure 8 illustrates several examples of the clas- 


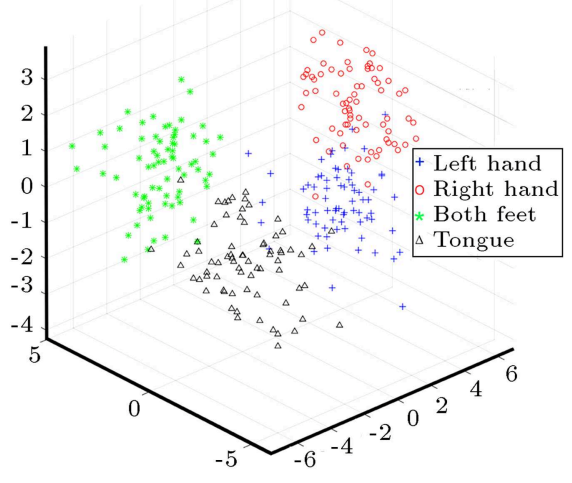

(a) BCI-competition-IV-S7

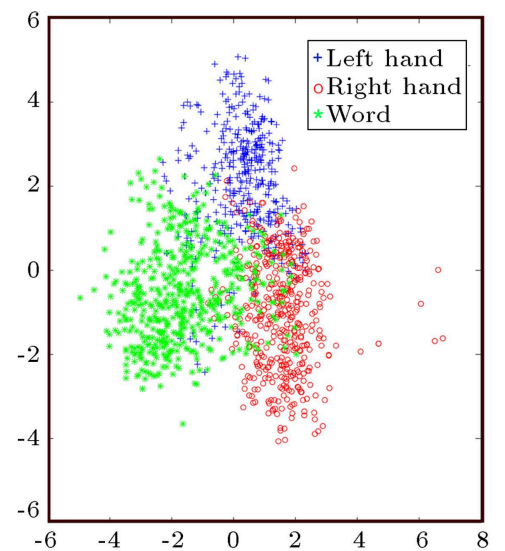

(b) BCI-competition-III-S1

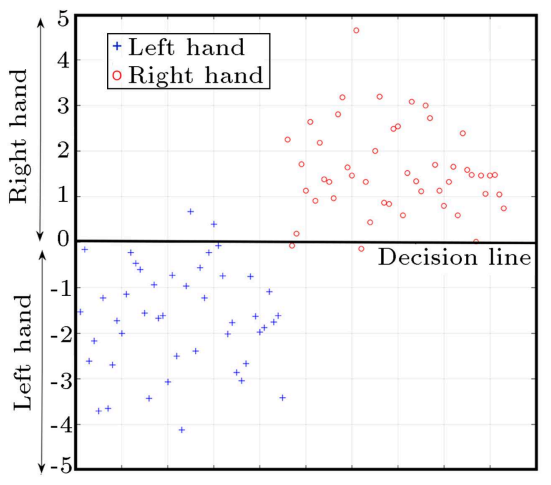

(c) Captured-signals-S4

Figure 8. Scatter of feature points output from LDA block. The dimension of LDA projection space is one less than the number of classes. As can be seen, the projected classes are well distinguished: (a) Imaginations of movement of left hand, right hand, both feet, and tongue projected in a three-dimensional LDA projection space, (b) imaginations of movement of left hand and right hand, and generation of words projected in a two-dimensional LDA projection space, and (c) imaginations of movement of left hand and right hand projected in a one-dimensional LDA projection space. For better visualization, a dummy dimension is added. (best viewed in color).

sified tasks by the proposed method. This figure illustrates the scatter of feature points output from LDA block. Figure 8(a) belongs to subject S7 of BCI competition IV. Because this dataset contains four classes, the dimension of feature vector from LDA output is of three. Figure 8(b) shows the first subject of BCI competition III, which has three classes. Thus, as it can be seen, features are two-dimensional. Figure $8(\mathrm{c})$ shows captured signals. In this dataset, there are two classes, and the dimension of feature vector of LDA output is one. However, for better visualization, the horizontal axis is added as a dummy dimension, which represents the index of samples. As is obvious in this figure, notably high separability between classes has been achieved as a result of optimum blocks used in this method.

\section{Signal processing concerns for hardware implementation}

In this section, issues relevant to the BCI system performance will be studied. Some parameters, such as the number of filter banks, the number of channels, the number of classes, and the number of features, affect the resulting hardware area. Thus, their values must be known before the hardware design. The dataset used for implementation is the third one (captured signals).

\subsection{Number of filter banks}

As previously mentioned, the EEG signals must pass the filter banks before applying the SCSSP algorithm. The number of banks should be efficient with regard to both the achieved accuracy and the hardware implementation cost. In this case, the number of filters is chosen to be four, so that these filters can cover alpha (8-12 Hz) and beta $(12-16 \mathrm{~Hz}, 16-20 \mathrm{~Hz}, 20-24 \mathrm{~Hz})$ frequency bands. The Chebyshev filter with order 6 is used for each filter path.

\subsection{Number of classes}

Another critical option is to choose the number of classes, influencing some parameters of LDA and SCSSP algorithms. In addition, it affects the classification design directly, because the basic SVM is only applicable to binary-class tasks. In order to use the SVM method for classification tasks with more than two classes, one-versus-all or one-versus-one approach can be used. In this study, since the hardware is tested for the third dataset, which contains only two classes, the number of classes of dataset is set to two.

\subsection{Number of channels}

Another parameter that affects the implementation is the number of channels. This parameter affects the clock rate of the DC-block, Laplacian filter, Chebyshev filter, and SCSSP module. The number of channels is set to 14 in the third dataset.

\subsection{Number of features}

The number of features, set to 40, influences the transfer matrix dimension of LDA used to reduce the number of features and SCSSP units, which generate the best features before applying LDA.

\section{Hardware implementation}

Herein, a two-class case is implemented as an instance of implementation. This implementation can be easily generalized to the case of more than two classes. Appli- 
cations of two-class case are very wide, such as moving the head of hospital bed up/down by thinking about movements of right/left hand. This can especially help patients with disabilities in moving body. There are also various applications in smart homes, where turning on and off the electrical devices can be performed by merely thinking about right and left hand movements. In addition, as mentioned in Section 2, recently in the literature, it has been proposed to code several tasks in a state machine using only the right- and left-hand signals [48].

In this work, solely, the testing phase of classification is implemented in hardware, as training can be performed beforehand and there is no need to implement it. The training phase can be done in software such as MATLAB or C language (MATLAB is used in this work), and the resulted coefficients can be saved and used for the test phase, which is implemented in hardware.

\subsection{Implementation of DC-block}

The first block in the proposed BCI system is the DCblock, with the structure, illustrated in Figure 9. As can be seen in this figure, it consists of one adder, one constant multiplier, and one subtractor. Because there are 14 parallel data channels that need to be filtered independently, there is a need for 14 parallel DC-block cores.

In such a situation, by means of applying the folding technique on the multiplier module (i.e., to design the DC-block with only one multiplier for all input channels), the silicon area minimization is enhanced. This achievement is obtained by sacrificing the throughput. However, because of the large sampling frequency of the recording device that is equal to $128 \mathrm{~Hz}$ for Emotiv ${ }^{\circledR}$ system, such a decrease in the throughput can be overlooked. As marked in Figure 9, the critical path of this core consists of only one multiplier and one adder, resulting in the operational frequency of up to $132 \mathrm{MHz}$, which is fast enough to handle such a low-frequency EEG signal.

\subsection{Implementation of Laplacian filter}

As stated in the previous sections, the Laplacian filter is based on the CAR algorithm, in which the mean of all channels is subtracted from all of them. The structure of this core is shown in Figure 10. There is one adder to compute the sum of all channels. The division of the output by the number of channels is realized through simple shift operations. In this way, a significant area and power can be saved, i.e., $\frac{1}{14}=\frac{1}{16}+$ $\frac{1}{128}+\frac{1}{1024}=0.0713$. As shown in Figure 10, the critical path of this module consists of two adders. Therefore, the operation frequency of this module can be up to 234 $\mathrm{MHz}$. The minimum speed of this module with respect to the sampling frequency of $128 \mathrm{~Hz}$ can be found based on the following equation: $\frac{14}{x}+\frac{1}{x}+\frac{14}{x}$ in which $x$ is the minimum limit of the operation frequency, which is $3718 \mathrm{~Hz}$.

\subsection{Implementation of Chebyshev filter}

Separable Common Spatio-Spectral Patterns (SCSSP) [30] are used to extract features of motor-imagery tasks. In this algorithm, input data goes through a bank of band-pass filters in the frequency ranges of 8$12 \mathrm{~Hz}$ ( $\alpha$ band) and $12-30 \mathrm{~Hz}$ ( $\beta$ band). We have used Chebyshev II band-pass filter of order 6 . The block diagram of this filter is illustrated in Figure 11. In this figure, $b_{i}$ and $a_{i}$ parameters are the nominator and denominator coefficients of this filter, respectively. It is important to note that this filter can be implemented by means of 12 multipliers (there are two constants and two zero coefficients). Each filter bank should be applied to all input channels simultaneously, and it ends up in a number of $168(12 \times 14)$ multipliers per filter bank, leading to $672(4 \times 168)$ multipliers with a bank of four filters, which is not feasible for implementation. In order to overcome this limitation, the folding technique is used between 14 input channels at each filter to reduce the total number of multipliers from 672 to 48 $(4 \times 12)$, resulting in a significant reduction in area and power consumption in this module. The critical path of this module consists of two multipliers and seven

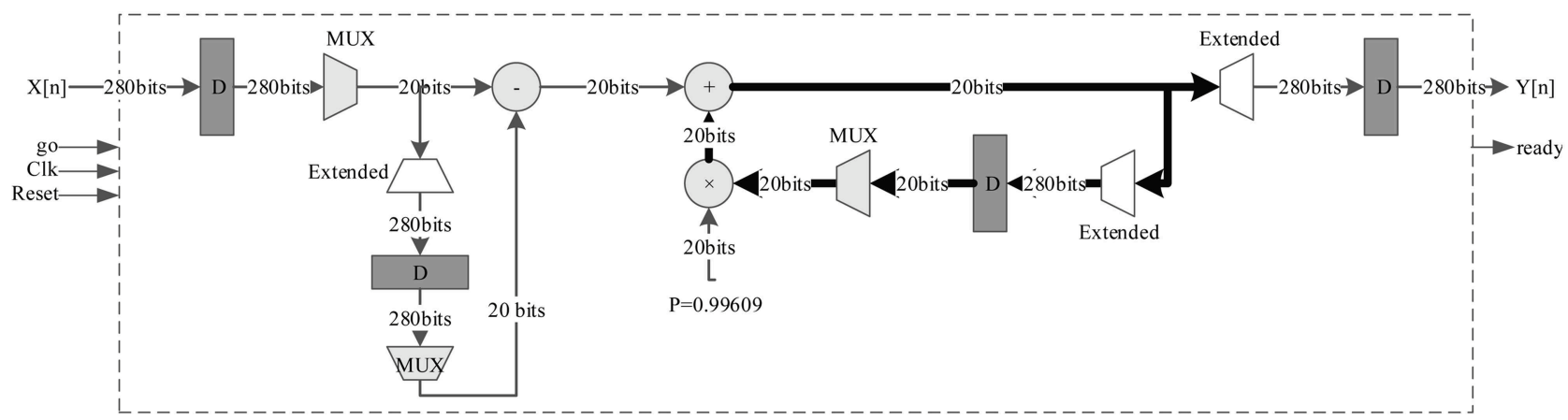

Figure 9. Block diagram of the proposed hardware implementation for DC-block. According to Eq. (1), we have $Y(z)\left(\rho z^{-1}-1\right)+X(z)\left(1-z^{-1}\right)=0$. Thus, $\rho Y[n-1]-Y[n]+X[n]-X[n-1]=0$. The left section of figure prepares $X[n]-X[n-1]$ and the right part creates $\rho Y[n-1]-Y[n]$. 


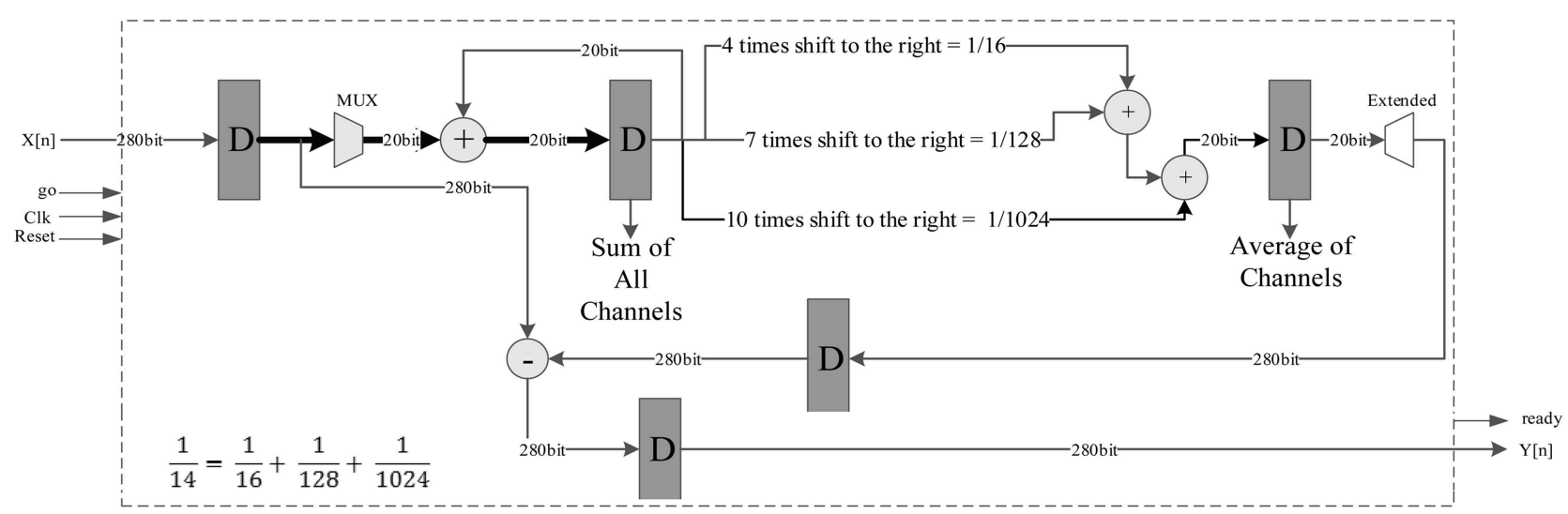

Figure 10. Block diagram of the proposed hardware implementation for Laplacian-Filter. Number of channels is 14 . The division $\frac{1}{14}$ in Eq. (3) is implemented using three simple shift-to-right actions: $\left(\frac{1}{16}+\frac{1}{128}+\frac{1}{1024}\right)$. The first adder sums up the whole channels. The subtraction is for subtracting average from every channel.

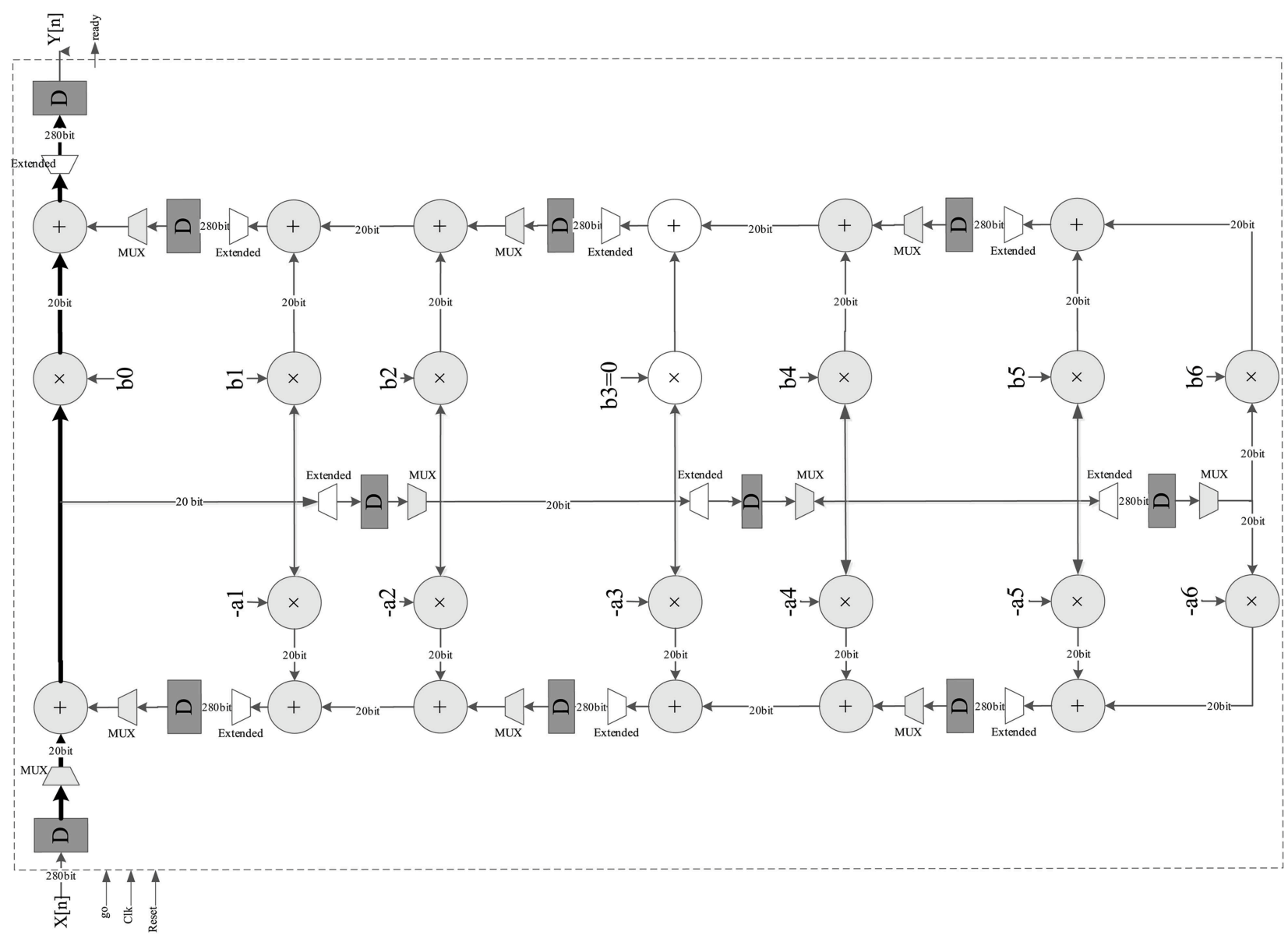

Figure 11. Block diagram of the proposed hardware implementation for Chebyshev. The transfer function of this filter can be written as $H(z)=\frac{Y(z)}{X(z)}=\frac{\sum_{i=0}^{6} b_{i} z^{-i}}{\sum_{i=0}^{6} a_{i} z^{-i}}$.

adders; however, it is improved by means of retiming technique to reduce this path to only one multiplier and two adders, resulting in maximum frequency of about $106 \mathrm{MHz}$. The latency of this module is 99 $(1+14 \times(6+1))$ clock cycles. Therefore, the minimum limit of the operational frequency in this module should be $12.3 \mathrm{kHz}(99 \times 128 \mathrm{~Hz})$.

\subsection{Implementation of SCSSP}

After applying the proposed band pass filters, the SCSSP [30] core should be applied to the filtered data, in order to extract corresponding features of motor-imagery tasks. The functional structure of this algorithm is summarized in Figure 12. As is shown in this figure, filtered vector $X$ consists of $4 \times 14$ elements 


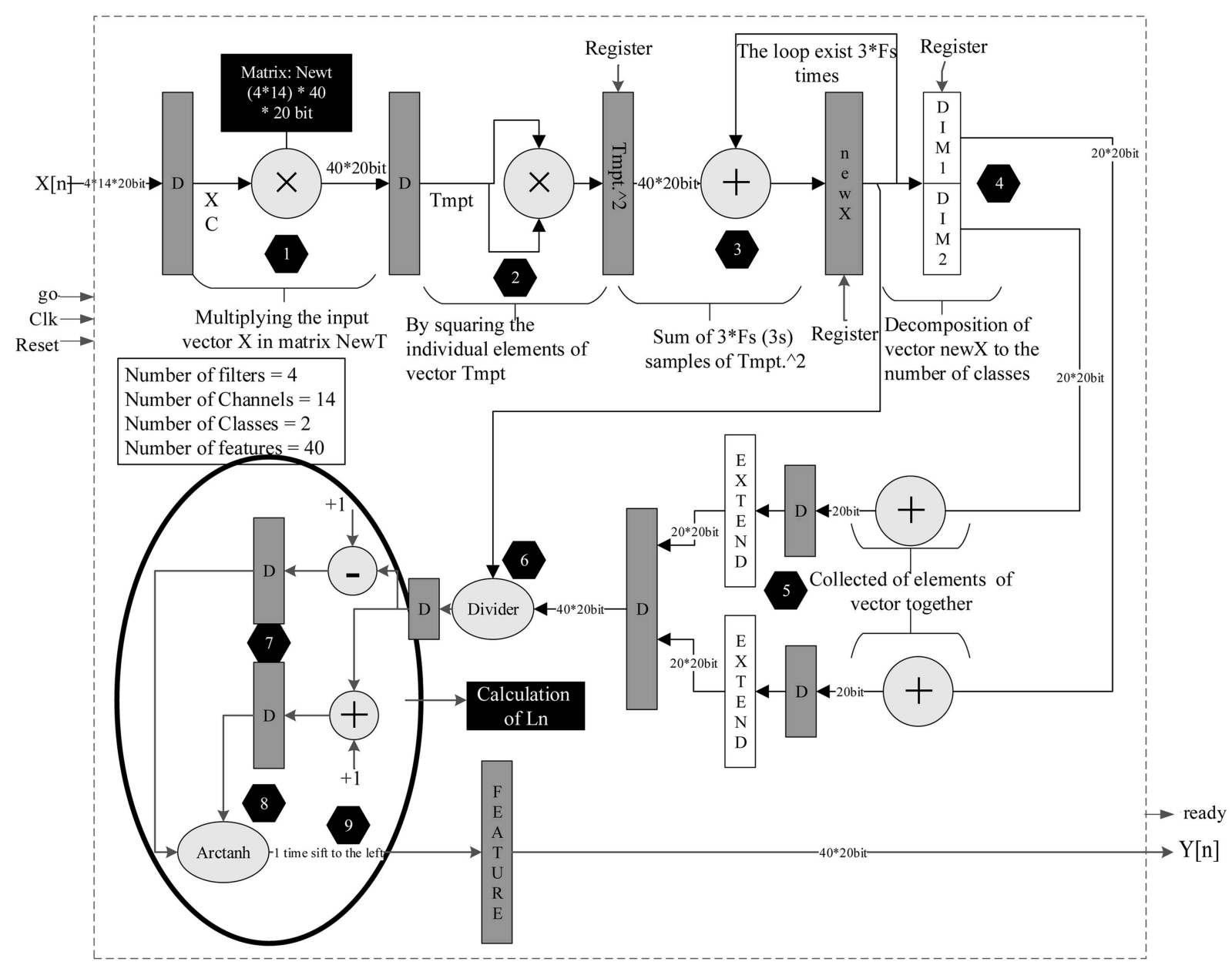

Figure 12. Block diagram of the proposed hardware implementation for SCSSP. Stage 1: Implementation of Eq. (8); Stages 2 and 3: implementation of $\operatorname{var}\left(y_{k}\right)$; Stages 4 and 5: implementation of $\sum_{k \in R} \operatorname{var}\left(y_{k}\right)$; Stage 6: implementation of $\frac{\operatorname{var}\left(y_{k}\right)}{\sum_{k \in R} \operatorname{var}\left(y_{k}\right)}$; Stages 7, 8, and 9: implementation of Eq. (9). Note that the eigenvalues and eigenvectors in this module are calculated formerly in the train phase though software.

from all 14 channels and 4 filters at a time instance. This vector should be multiplied by the "NewT" matrix that consists of 40 sub-matrices with size of $4 \times 14$ (there are 40 features), whose elements are determined during the train phase of the experiment. The matrix "NewT" is $\left(\mathbf{w}_{R, j[k]} \otimes \mathbf{w}_{L, i[k]}\right)^{T}$, where $\mathbf{w}_{R, j[k]}$ and $\mathbf{w}_{L, i[k]}$ are the eigenvectors in $W_{R}$ and $W_{L}$ corresponding to $\lambda_{R}^{j[k]}$ and $\lambda_{L}^{i[k]}$, explained in SCSSP section. The result is named "Tmpt" vector, whose elements are squared to construct "Tmpt2" vector. This procedure is repeated after receiving the next sample whose "Tmpt2" vector is added to the previous "Tmpt2". This job is repeated for $3 \times f_{s}(128 \mathrm{~Hz})$ times. After that, the new "Tmpt2" vector is decomposed to 2 parts due to the number of classes. Each part of the vector consists of 20 elements. The next step is to sum up these elements so that each part of these vectors is divided by its corresponding sum result. The implementation of the logarithmic function is also implemented by the following equivalency:

$$
\operatorname{Ln}(x)=2 \operatorname{Arctanh}\left(\frac{x-1}{x+1}\right) .
$$

An overview of different stages in SCSSP algorithm is marked by means of black hexagons in Figure 12. Each stage is a sub-module and designed to dictate a total critical path of one adder and one multiplier for the whole design, which was made possible using an effective pipelining technique specifically in the divider and Arctanh operations, in addition to the folding and retiming techniques used in the design. These efforts resulted in an effective high-speed feature extraction operating at $102 \mathrm{MHz}$ clock speed. The minimum speed of the operational frequency in this module is $560 \mathrm{kHz}$.

\subsection{Implementation of normalization}

After feature extraction, the features should be normalized. To reach this goal, test features should be subtracted from the average of train features and, then, divided into standard deviation of train features. 


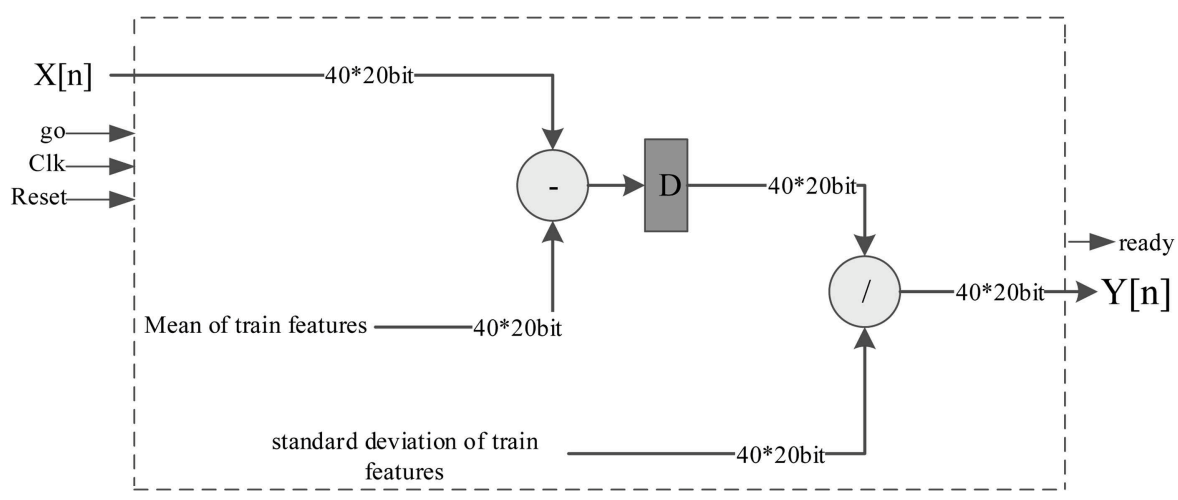

Figure 13. Block diagram of the proposed hardware implementation for normalization. Notice that the mean and standard deviation of training data (Eqs. (10) and (11)) are calculated erstwhile in the software and are used here as constant vectors. This figure illustrates the implementation of Eq. (12).

The block diagram of normalization is illustrated in Figure 13. The latency of this module is 40 clock periods. The number of clocks taken to generate output for each feature is 27 . Since there are 40 features, it can be concluded that the number of total clocks for this part is $40 \times 27+40=1120$. Therefore, the minimum operational frequency of this module is $1120 \times 128=143360 \mathrm{~Hz}$. The maximum speed of this module can be up to $267 \mathrm{MHz}$.

\subsection{Implementation of $M I$}

After normalization, appropriate features should be chosen. Fortunately, this part does not require any hardware. In a training stage, the indices of features are determined. Therefore, according to the index, the corresponding elements of the feature vectors are selected in a test stage. To explain better, the maximum number of features is set to 40 , which is found to work for all subjects. In the training phase, mutual information module finds the best indices and the number of features for every subject. This training is performed in software, as explained previously. Then, those elements of transformation matrix (LDA module), corresponding with those numbers of features which are not chosen by mutual information, will be replaced with zeros (masking). Therefore, those numbers of acceptable features are only calculated in LDA module in the testing phase. In the testing phase, a masking process is performed merely in LDA module in order to select the best-found features out of 40 features. Note that, consequently, altering the number of features does not affect the hardware at all. In conclusion, no hardware resources are required to implement mutual information module since the features are selected in the training phase.

\subsection{Implementation of $L D A$}

After choosing the best features, their dimension should be reduced. This reduction can be performed by means of the LDA algorithm, which was discussed previously. In this algorithm, at the train stage, a key matrix is generated. If eigenvectors of this matrix consist of $d$ elements (here, $d=40$ ), then the dimension of this matrix must be $(C-1) \times d$, where $C$ is the number of classes. Hence, after multiplying the matrix of selected features by the eigenvectors, the dimension of output vector will be $C-1$. Herein, due to the classification of left- and right-hand movement motorimagery tasks, $C$ is equal to 2 and the output is a scalar number. The block diagram of this multiplication is depicted in Figure 14. In this module, the critical path consists of one multiplier and one adder, and the maximum speed of this module can be up to 203 $\mathrm{MHz}$. The latency of this module is 40 clock periods; therefore, the minimum operational frequency of this module should be $5128(40 \times 128) \mathrm{Hz}$.

\subsection{Implementation of $S V M$}

The last core in the data flow is the classifier. This core is designed based on the Linear-SVM algorithm. The main reason for choosing the linear classifier is its simplicity and efficiency in hardware implementation. The block diagram of this core is shown in Figure 15. Based on this figure, the critical path consists of just one multiplier. Therefore, the maximum operational frequency of this module is around $211 \mathrm{MHz}$. The latency of this module is 2 clock periods; consequently, the minimum limit of the speed of this core is $256(2 \times 128) \mathrm{Hz}$.

It is also worth mentioning that implementing non-linear SVM requires adding a non-linear function block to obtain the non-linear function of the dot product between the support vectors and the test vector.

\section{Trade-off of accuracy and hardware resources}

As mentioned before, linear SVM is preferred to Bayesian classifier in this work. The detailed comparison of these two classifiers in terms of performance results and hardware resources is reported in Table 2. 


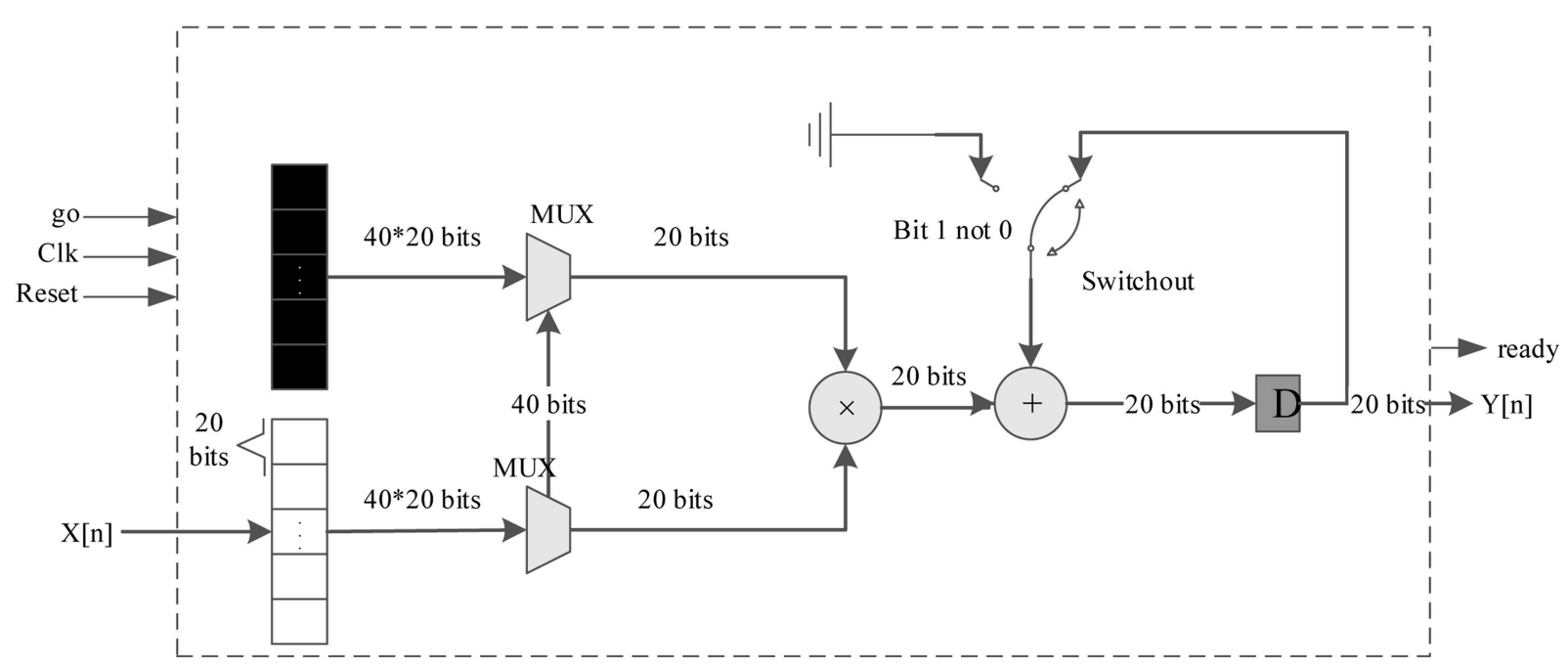

Figure 14. Block diagram of the proposed hardware implementation for LDA. The matrix of eigenvectors has dimension of $(C-1) \times 40=1 \times 40$, and the input vector $X[n]$ is a $40 \times 1$ vector. By multiplying these two vectors, a scalar output is obtained. The multiplication of these vectors is the same as inner product, and the multiplications of elements are accumulated using an adder. Note that the matrix of eigenvectors is obtained formerly through software.

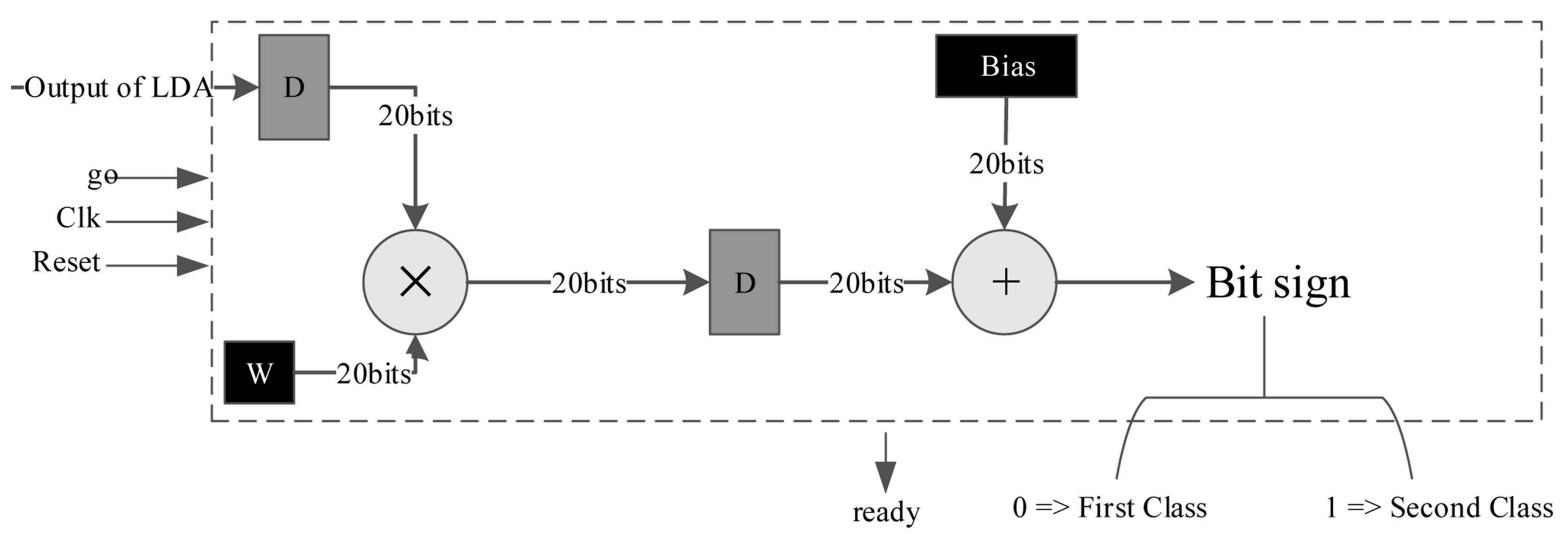

Figure 15. Block diagram of the proposed hardware implementation for Linear SVM. The decision function of SVM is $\operatorname{sign}\left(w^{T} x+b\right)$ [68], where $w^{\prime}$ 's are the weights of SVM, $b$ is the bias, and here $x$ is the output of the previous stage, which is LDA.

Table 2. Comparison of SVM and Bayesian classifiers in terms of performance results and hardware resources.

\begin{tabular}{|c|c|c|c|c|c|c|c|c|c|c|c|}
\hline \multicolumn{12}{|c|}{ Performance results } \\
\hline & & S1 & S2 & S3 & $\mathrm{S} 4$ & S5 & S6 & S7 & S8 & S9 & Average \\
\hline \multirow{2}{*}{$\begin{array}{l}\text { BCI competition III } \\
\text { (Dataset V, } 3 \text { classes) }\end{array}$} & SVM & $86.60 \%$ & $78.00 \%$ & $56.00 \%$ & $x$ & $x$ & & $x$ & $\times$ & $x$ & $73.53 \%$ \\
\hline & Bayesian & $86.60 \%$ & $78.00 \%$ & $61.00 \%$ & $x$ & $x$ & $\times$ & $x$ & $\times$ & $\times$ & $75.20 \%$ \\
\hline BCI competition & SVM & $79.20 \%$ & $56.30 \%$ & $87.50 \%$ & $63.90 \%$ & $41.00 \%$ & $46.90 \%$ & $81.90 \%$ & $76.00 \%$ & $72.20 \%$ & $67.21 \%$ \\
\hline IV(Dataset 2a, 4 classes) & Bayesian & $80.55 \%$ & $60.10 \%$ & $87.50 \%$ & $67.70 \%$ & $42.70 \%$ & $46.20 \%$ & $82.30 \%$ & $78.10 \%$ & $74.00 \%$ & $68.79 \%$ \\
\hline \multirow{2}{*}{$\begin{array}{c}\text { BCI competition IV } \\
\text { (Dataset } 2 \mathrm{a}, 2 \text { classes) }\end{array}$} & SVM & $91.67 \%$ & $59.72 \%$ & $95.83 \%$ & $77.08 \%$ & $67.36 \%$ & $69.44 \%$ & $78.47 \%$ & $97.22 \%$ & $88.19 \%$ & $80.55 \%$ \\
\hline & Bayesian & $90.27 \%$ & $59.72 \%$ & $95.83 \%$ & $78.47 \%$ & $67.36 \%$ & $69.44 \%$ & $77.08 \%$ & $97.22 \%$ & $90.00 \%$ & $80.59 \%$ \\
\hline \multirow[t]{2}{*}{ Captured signals } & SVM & $93.60 \%$ & $93.60 \%$ & $96.80 \%$ & $83.90 \%$ & $71.00 \%$ & $71.00 \%$ & $58.00 \%$ & $87.10 \%$ & $\times$ & $81.87 \%$ \\
\hline & Bayesian & $93.60 \%$ & $93.60 \%$ & $96.80 \%$ & $83.90 \%$ & $71.00 \%$ & $71.00 \%$ & $58.00 \%$ & $87.10 \%$ & $\times$ & $81.87 \%$ \\
\hline \multicolumn{12}{|c|}{ Hardware resources } \\
\hline & & $\begin{array}{l}\text { Add/ } \\
\text { Sub }\end{array}$ & Multp & Divider & Arctan & $\begin{array}{l}\text { Sinh/ } \\
\text { Cosh }\end{array}$ & $\begin{array}{l}\text { Latency } \\
\text { (clocks) }\end{array}$ & $\begin{array}{l}\text { Power } \\
(\mathrm{mW})\end{array}$ & LUTs & Registers & $x$ \\
\hline \multirow{2}{*}{ Captured signals } & SVM & 1 & 1 & 0 & 0 & 0 & 2 & 4.69 & 63 & 21 & $x$ \\
\hline & Bayesian & 3 & 3 & 0 & 0 & 1 & 59 & 20.44 & 172 & 211 & $x$ \\
\hline
\end{tabular}


Table 3. The settings of points (experiments) in the Pareto optimal graph shown in Figure 16.

\begin{tabular}{cccccccccc}
\hline Point & $\begin{array}{c}\text { DC } \\
\text { block }\end{array}$ & Laplacian & SCSSP & Normalization & MI & LDA & $\begin{array}{c}\text { SVM/ } \\
\text { Bayesian }\end{array}$ & $\begin{array}{c}\text { Accuracy } \\
(\%)\end{array}$ & $\begin{array}{c}\text { Power } \\
(\mathbf{m W})\end{array}$ \\
\hline 1 & $\sqrt{ }$ & $\sqrt{ }$ & $\sqrt{ }$ & $\sqrt{ }$ & $\sqrt{ }$ & $\sqrt{ }$ & SVM & $81.9 \%$ & 83.00 \\
2 & $\sqrt{ }$ & $\times$ & $\sqrt{ }$ & $\sqrt{ }$ & $\sqrt{ }$ & $\sqrt{ }$ & SVM & $75.5 \%$ & 85.34 \\
3 & $\times$ & $\sqrt{ }$ & $\sqrt{ }$ & $\sqrt{ }$ & $\sqrt{ }$ & $\sqrt{ }$ & SVM & $77.9 \%$ & 79.49 \\
4 & $\sqrt{ }$ & $\sqrt{ }$ & $\sqrt{ }$ & $\times$ & $\sqrt{ }$ & $\sqrt{ }$ & SVM & $77.5 \%$ & 67.80 \\
5 & $\sqrt{ }$ & $\times$ & $\sqrt{ }$ & $\times$ & $\sqrt{ }$ & $\sqrt{ }$ & SVM & $75.2 \%$ & 70.14 \\
6 & $\times$ & $\sqrt{ }$ & $\sqrt{ }$ & $\times$ & $\sqrt{ }$ & $\sqrt{ }$ & SVM & $76.0 \%$ & 73.65 \\
7 & $\times$ & $\times$ & $\sqrt{ }$ & $\sqrt{ }$ & $\sqrt{ }$ & $\sqrt{ }$ & SVM & $75.5 \%$ & 78.32 \\
8 & $\times$ & $\times$ & $\sqrt{ }$ & $\times$ & $\sqrt{ }$ & $\sqrt{ }$ & SVM & $75.2 \%$ & 75.99 \\
9 & $\sqrt{ }$ & $\sqrt{ }$ & $\sqrt{ }$ & $\sqrt{ }$ & $\sqrt{ }$ & $\sqrt{ }$ & Bayesian & $80.8 \%$ & 90.01 \\
10 & $\sqrt{ }$ & $\times$ & $\sqrt{ }$ & $\sqrt{ }$ & $\sqrt{ }$ & $\sqrt{ }$ & Bayesian & $76.7 \%$ & 94.69 \\
11 & $\times$ & $\sqrt{ }$ & $\sqrt{ }$ & $\sqrt{ }$ & $\sqrt{ }$ & $\sqrt{ }$ & Bayesian & $76.4 \%$ & 87.68 \\
12 & $\sqrt{ }$ & $\sqrt{ }$ & $\sqrt{ }$ & $\times$ & $\sqrt{ }$ & $\sqrt{ }$ & Bayesian & $77.1 \%$ & 80.66 \\
13 & $\sqrt{ }$ & $\times$ & $\sqrt{ }$ & $\times$ & $\sqrt{ }$ & $\sqrt{ }$ & Bayesian & $74.0 \%$ & 86.51 \\
14 & $\times$ & $\sqrt{ }$ & $\sqrt{ }$ & $\times$ & $\sqrt{ }$ & $\sqrt{ }$ & Bayesian & $73.9 \%$ & 78.32 \\
15 & $\times$ & $\times$ & $\sqrt{ }$ & $\sqrt{ }$ & $\sqrt{ }$ & $\sqrt{ }$ & Bayesian & $76.7 \%$ & 75.98 \\
16 & $\times$ & $\times$ & $\sqrt{ }$ & $\times$ & $\sqrt{ }$ & $\sqrt{ }$ & Bayesian & $74.0 \%$ & 78.32 \\
\hline
\end{tabular}

As can be seen in this table, the Bayesian classifier slightly outperforms linear SVM in accuracy, while its hardware resource usage is significantly higher and is also much slower than SVM, making it not suitable for motor imagery systems in which fast decision-making is crucial. The very small improvement in accuracy while causing a significant increase in hardware usage and latency is not encouraging. Moreover, note that power is one of the main concerns in BCI systems fulfilled by choosing linear SVM over Bayesian classifier. Nevertheless, one can prefer better accuracy if hardware usage is not an important issue.

For showing the trade-off between accuracy and hardware resources better, different permutations of settings are experimented by excluding/including the parts from/in the proposed system. The classification accuracy as well as the consumed power of the experiments are reported by a Pareto optimal graph illustrated in Figure 16. The settings of different experiments (points) in this figure are listed in Table 3. Experiments 1 and 9 in this Pareto optimal graph are the experiments detailed in Table 2. The Pareto optimal graph clearly shows that there exists a trade-off between classification accuracy and hardware efficiency, and some settings are the best ones among the possible settings. As can be seen in Figure 16, experiments 1,3 , and 4 are the Pareto optimal points from which the setting of experiment 1 is chosen as the final setting in this paper, because accuracy is important in our goal.

\section{Hardware results}

In order to capture, analyze, and process the captured data, the following interfaces and tools are used. The

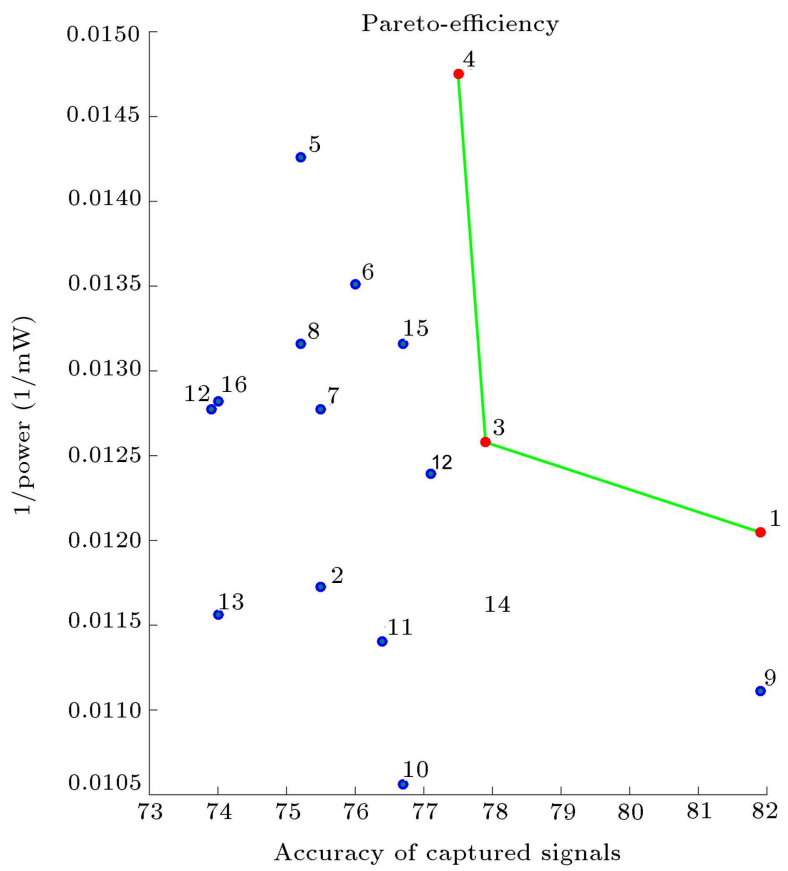

Figure 16. The Pareto optimal graph for reporting the trade-off between accuracy and power. The settings of the points are listed in Table 3. The red points connected with the green lines are the Pareto optimal points.

utilized evaluation board is Virtex-6 FPGA ML605 Evaluation Platform to which the input data is sent via an Ethernet port. The output signal is tracked and analyzed by Xilinx ChipScope software. The synthesis and analysis of HDL designs are performed using ISE Xilinx 14.5 software. The results of fixed-point numbers show that 20 bits are enough for each value of every channel in a way that 11 bits are considered for the decimal part, 8 bits for the integer part, and one 
last bit for the sign bit. Thus, the number of bits for one test vector is $280(14$ channels $\times 20)$. A new test vector is processed every $0.0078 \mathrm{~s}\left(f_{s}=128 \mathrm{~Hz}\right)$. Since each person requires three seconds to perform a given task (see Figure 3 ), then the number of test vectors for each experiment is equal to $384(3 \times 128)$. Each person performs 30 experiments including 15 experiments for the first class and 15 experiments for the second class (in our captured dataset).

Retiming and folding techniques were used by sacrificing the speed, which is justifiable because of low sampling frequency of signal recording. Final structure satisfies maximum frequency of $102 \mathrm{MHz}$ and minimum frequency of $560 \mathrm{kHz}$, according to Table 4 . Table 4 shows the number of Adders, Subtractors, Multipliers, Dividers, and Arctan modules used in the proposed BCI, showing the importance of using folding and retiming techniques. Note that, for multiplications, Xilinx multiplication cores (LogiCORE IP Multiplier v11.2), which are optimized according to inputs of the core, are utilized. The hardware latency and power of each section and the total framework are also reported in Table 4. Moreover, as seen in Table 4, a wide range of allowed frequencies is provided in this proposed system, helping to increase frequency and have a satisfying and acceptable total latency even in a large number of channels.

The hardware resource usage is reported in Table 5 . It can be seen that the total power of this work, i.e., $83.90 \mathrm{~mW}$, is much less than the total power in [48], which is $1.067 \mathrm{~W}$. As reported in this table, the proposed system outperforms [47] and [48] significantly in terms of the number of LUTs, the number of block RAM/FIFO, the total latency, and the utilized power.

Table 4. Features of sections of the framework. The first two columns report the valid operational frequency of the proposed BCI. The next four columns represent the detailed resources utilized in different sections. The last two columns display latency and power, respectively. Note that the total latency is the spent time started at the end of motor imagery (which is the 6th second according to Figure 3 ) and ended when prediction becomes ready. In this table, $N$ and $D$ respectively denote the number of channels and features.

\begin{tabular}{|c|c|c|c|c|c|c|c|c|}
\hline & $\begin{array}{l}\text { Min. } \\
\left(\mathbf{H z}_{\mathrm{z}}\right)\end{array}$ & $\begin{array}{c}\text { Max. } \\
(\mathbf{H z})\end{array}$ & $\begin{array}{c}\text { Add/ } \\
\text { Sub }\end{array}$ & Multp & Divider & Arctan & Latency $^{1}$ & $\begin{array}{l}\text { Power } \\
(\mathrm{mW})\end{array}$ \\
\hline DC-block & 1792 & $132 \mathrm{M}$ & 2 & 1 & 0 & 0 & $14=N$ & 7.44 \\
\hline Laplacian filter & 3718 & $234 \mathrm{M}$ & 4 & 0 & 0 & 0 & $18=N+4$ & 1.70 \\
\hline Chebyshev filter & $12.3 \mathrm{~K}$ & $106 \mathrm{M}$ & $4 \times 11$ & $4 \times 12$ & 0 & 0 & $99=7 N+1$ & 54.15 \\
\hline SCSSP & $560 \mathrm{~K}$ & $102 \mathrm{M}$ & 5 & 2 & 0 & 1 & $4375=4 N D+50 D+135$ & 22.04 \\
\hline Normalization & 143360 & $267 \mathrm{M}$ & 1 & 0 & 1 & 0 & $1120=27 N+40$ & 12.61 \\
\hline MI & - & - & 0 & 0 & 0 & 0 & 0 & 0 \\
\hline LDA & 5128 & $203 \mathrm{M}$ & 1 & 1 & 0 & 0 & $40=D$ & 7.99 \\
\hline SVM & 256 & $211 \mathrm{M}$ & 1 & 1 & 0 & 0 & 2 & 4.69 \\
\hline BCI (total system) & $560 \mathrm{~K}$ & $102 \mathrm{M}$ & 58 & 53 & 1 & 1 & $5668=182+36 N+51 D+4 N D$ & 83.90 \\
\hline
\end{tabular}

${ }^{1}$ Numbers of this column are in clock numbers. The minimum and maximum clock frequencies are reported in the two first columns. Notice that identical clock frequency $(560 \mathrm{kHz} \leq f \leq 102 \mathrm{MHz})$ is used in the sections of final setup. The latency can be obtained in milliseconds according to selected clock frequency (10 milli-seconds in the worst case and 55.5 micro-seconds in the best case).

Table 5. Hardware resource usage. The proposed framework outperforms [47] in number of lookup-tables and block RAM/FIFO and cost of a few additional DSP blocks. It also outperforms [48] in number of block RAM/FIFO.

\begin{tabular}{cccccc}
\hline & LUTs & $\begin{array}{c}\text { Block } \\
\text { RAM/FIFO }\end{array}$ & $\begin{array}{c}\text { DSP } \\
\text { block }\end{array}$ & $\begin{array}{c}\text { Latency } \\
(\mathbf{m s})^{\mathbf{1}}\end{array}$ & $\begin{array}{c}\text { Power } \\
(\mathbf{W})^{\mathbf{1}}\end{array}$ \\
\hline This work & $\mathbf{1 1 , 3 1 1}$ & $\mathbf{6 5 , 5 3 6}$ & $\mathbf{2 4}$ & $\leq \mathbf{1 0}$ & $\mathbf{0 . 0 8 3}$ \\
{$[47]$} & 17,281 & 557,332 & $\mathbf{4}$ & 57776.066 & - \\
{$[48]$} & 27,906 & 818,624 & 644 & 399 & 1.067 \\
\hline
\end{tabular}

${ }^{1}$ The numbers of channels in the hardware implementation are 14, 22, and 22

in this work, [47], and [48], respectively, and the reported latency and power in this table are set according to these settings. However, note that, according to Table 4, latency of the proposed system would be $\leq 6534 \times(1 / 560 \mathrm{kHz}) \leq 11.66$ ms for $N=22$ channels much better than in [47] and [48]. In addition, as explained in paragraph 3 of Section 9, the hardware is not altered significantly by changing number of channels; therefore, power does not significantly change, too. 
It outperforms [48] in the number of DSP blocks, too. The reason of outperforming [48] in the number of DSP blocks and multipliers is having filters with order six, while the order of filters increases significantly (to, e.g., 221 and 442) to achieve higher accuracy. On the other hand, the proposed system outperforms [48] in terms of block RAM/FIFO which is because most of the tuned and trained parameters saved in our system are scalars (e.g., in SVM) or vectors (e.g., in LDA) and merely one matrix saved for SCSSP. However, more matrices are required to be saved in [48], which are the matrices needed for CSP section and covariance matrix in Mahalanobis distance. Moreover, folding and retiming techniques help our system have better power consumption and area. Paper [47], although has the lower performance rate $(72 \%)$ on BCI Competition IV dataset (2 classes), its latency is not completely proper for practical applications.

Note that, in the proposed system, changing the number of channels does not alter the utilized hardware resources too much because normalization, SVM, and LDA structures are independent of the number of the channels, and merely some wires and bits in registers are added in DC-block, Laplacian filter, Chebyshev filter, and SCSSP. Consequently, the number of RAM/FIFO and DSP blocks do not change, while the number of LUTs blocks increases a little and not significantly.

\section{Conclusion}

This paper proposed an efficient hardware implementation for BCI systems. The proposed system consists of preprocessing, feature extraction, feature selection, feature reduction, and classification stages. Software simulations and hardware limitations guided the method to choose DC-block and surface Laplacian as preprocessing, SCSSP as feature extractor, MI as feature selector, LDA as feature reduction method, and SVM as classifier. Results determined the proper performance of the proposed system, compared to other reported results on the used datasets $(67.2 \%$ for BCI competition IV, $73.54 \%$ for BCI competition III, and $81.9 \%$ for captured signals). The system was also efficiently implemented and tested on a FPGA hardware platform. The minimum frequency of BCI system was $560 \mathrm{kHz}$. Moreover, because of using Folding and Retiming techniques, the number of resources (LUTs and block RAM/FIFO) decreased dramatically, on cost of a few additional DSP blocks.

Blinking can have destructive impact on the obtained accuracy. By using an appropriate EOG detection during the mental task, it is possible to increase the signal-to-noise ratio and, thus, accuracy. Moreover, in all these experiments, it was known when the subjects were going to do the mental task.
Predicting when subjects are going to think about the task by extracting ERD/ERS features can be added to the proposed framework (see Section 3.3 of [69]).

\section{References}

1. Wolpaw, J.R., Birbaumer, N., McFarland, D.J., Pfurtscheller, G., and Vaughan, T.M. "Braincomputer interfaces for communication and control", Clin. Neurophysiol., 113(6), pp. 767-791 (2002).

2. Duchowski, A.T., Eye Tracking Methodology: Theory and Practice, Springer International Publishing AG, 3rd Ed., ISBN 978-3-319-57881-1, ISBN 978-3319-57883-5 (eBook) (2017). DOI 10.1007/978-3-319$57883-5$

3. Ferracuti, F., Freddi, A., Iarlori, S., Longhi, S., and Peretti, P. "Auditory paradigm for a p300 BCI system using spatial hearing", In Intelligent Robots and Systems (IROS), 2013 IEEE/RSJ International Conference on, IEEE, pp. 871-876 (2013).

4. Káthner, I., Ruf, C.A., Pasqualotto, E., Braun, C., Birbaumer, N., and Halder, S. "A portable auditory P300 brain-computer interface with directional cues", Clinical Neurophysiology, 124(2), pp. 327-338 (2013).

5. Kim, D.-W., Hwang, H., Lim, J.-H., Lee, Y.-H., Jung, K.-Y., and Im, C.-H. "Classification of selective attention to auditory stimuli: toward vision-free braincomputer interfacing", Journal of Neuroscience Methods, 197(1), pp. 180-185 (2011).

6. Pfurtscheller, G., Brunner, C., Schlögl, A., and Da Silva, F.H.L. "Mu rhythm (de) synchronization and EEG single-trial classification of different motor imagery tasks", NeuroImage, 31(1), pp. 153-159 (2006).

7. Khurana, K., Gupta, P., Panicker, R.C., and Kumar, A. "Development of an FPGA-based real-time P300 speller", 22nd International Conference on Field Programmable Logic and Applications (FPL), Oslo, pp. 551-554 (2012).

8. Xu, B., Song, A., and Wu, J. "Algorithm of imagined left-right hand movement classification based on wavelet transform and AR parameter model", Bioinformatics and Biomedical Engineering, The 1st International Conference on. IEEE (2007).

9. Schlögl, A., The Electroencephalogram and the Adaptive Autoregressive Model: Theory and Applications, Germany: Shaker (2000).

10. Pfurtscheller, G., Neuper, C., Schlogl, A., and Lugger, K. "Separability of EEG signals recorded during right and left motor imagery using adaptive autoregressive parameters", in IEEE Transactions on Rehabilitation Engineering, 6(3), pp. 316-325 (Sept. 1998).

11. Schlögl, A., Lugger, K., and Pfurtscheller, G. "Using adaptive autoregressive parameters for a braincomputer-interface experiment", Proceedings 19th International Conference IEEE/EMBS (1997).

12. Dharmasena, S., Lalitharathne, K., Dissanayake, K., Sampath, A., and Pasqual, A. "Online classification of 
imagined hand movement using a consumer grade EEG device", Industrial and Information Systems (ICIIS), 8th IEEE International Conference on. IEEE (2013).

13. Alomari, M.H., Samaha, A., and AlKamha, K. "Automated classification of $\mathrm{L} / \mathrm{R}$ hand movement $\mathrm{EEG}$ signals using advanced feature extraction and machine learning", International Journal of Advanced Computer Science and Applications, 4(6), pp. 207-212 (2013).

14. Xu, Q., Zhou, H., Wang, Y., and Huang, J. "Fuzzy support vector machine for classification of EEG signals using wavelet-based features", Medical Engineering \& Physics, 31(7), pp. 858-865 (2009).

15. Ebrahimpour, R., Babakhani, K., and MohammadNoori, M. "EEG-based motor imagery classification using wavelet coefficients and ensemble classifiers", Artificial Intelligence and Signal Processing (AISP), 16th CSI International Symposium on. IEEE (2012).

16. Carrera-Leon, O., Ramirez, J.M., Alarcon-Aquino, V., Baker, M., D'Croz-Baron, D., and Gomez-Gil, P. "A motor imagery $\mathrm{BCI}$ experiment using wavelet analysis and spatial patterns feature extraction", Engineering Applications (WEA), 2012 Workshop on. IEEE (2012).

17. Jahankhani, P., Kodogiannis, V., and Revett, K. "EEG signal classification using wavelet feature extraction and neural networks", John Vincent Atanasoff 2006 International Symposium on Modern Computing (JVA'06), IEEE (2006).

18. Subasi, A. "Automatic recognition of alertness level from EEG by using neural network and wavelet coefficients", Expert Systems with Applications, Elsevier, 28(4), pp. 701-711 (2015).

19. Ting, W., et al. "EEG feature extraction based on wavelet packet decomposition for brain computer interface", Measurement, Elsevier, 41(6), pp. 618-625 (2008).

20. Subasi, A. "EEG signal classification using wavelet feature extraction and a mixture of expert model", Expert Systems with Applications, 32(4), pp. 10841093 (2007).

21. Lotte, F., Guan, C., and Ang, K.K. "Comparison of designs towards a subject-independent brain-computer interface based on motor imagery", Annual International Conference of the IEEE Engineering in Medicine and Biology Society, Minneapolis, MN, pp. 4543-4546 (2009).

22. Ramoser, H., Mller-Gerking, J., and Pfurtscheller, G. "Optimal spatial filtering of single trial EEG during imagined hand movement", IEEE Transactions on Rehabilitation Engineering, 8(4), pp. 441-446 (2000).

23. Suk, H.-I. and Lee, S.-W. "A probabilistic approach to spatiospectral filters optimization in brain-computer interface", International Conference on Systems, Man, and Cybernetics (SMC), IEEE, pp. 19-24 (2011).

24. Lemm, S., Blankertz, B., Curio, G., and Mller, K. "Spatio-spectral filters for improving the classification of single trial EEG", IEEE Transactions on Biomedical Engineering, 52(9), pp. 1541-1548 (2005).

25. Dornhege, G., Blankertz, B., Krauledat, M., Losch, F., Curio, G., and Muller, K.R. "Combined optimization of spatial and temporal filters for improving braincomputer interfacing", IEEE Transactions on Biomedical Engineering, 53(11), pp. 2274-2281 (2006).

26. Wu, W., Gao, X., Hong, B., and Gao, S. "Classifying single-trial EEG during motor imagery by iterative spatio-spectral patterns learning (ISSPL)", IEEE Transactions on Biomedical Engineering, 55(6), pp. 17331743 (2008).

27. Zhang, H., Chin, Z.Y., Ang, K.K., Guan, C., and Wang, C. "Optimum spatio-spectral filtering network for brain-computer interface", IEEE Transactions on Neural Networks, 22(1), pp. 52-63 (2011).

28. Ang, K.K., Chin, Z.Y., Wang, C., Guan, C., and Zhang, H. "Filter bank common spatial pattern algorithm on BCI competition IV datasets $2 \mathrm{a}$ and $2 \mathrm{~b}$ ", Frontiers in Neuroscience, 6(39), pp. 1-9 (2012).

29. Ang, K.K. and Guan, C. "EEG-based strategies to detect motor imagery for control and rehabilitation", IEEE Transactions on Neural Systems and Rehabilitation Engineering, 25(4), pp. 392-401 (2017).

30. Aghaei, A.S., Mahanta, M.S., and Plataniotis, K.N. "Separable common spatio-spectral pattern algorithm for classification of EEG signals", Acoustics, Speech and Signal Processing (ICASSP), 2013 IEEE International Conference on. IEEE (2013).

31. Lotte, F. and Guan, C. "Regularizing common spatial patterns to improve BCI designs: unified theory and new algorithms", IEEE Transactions on Biomedical Engineering, 58(2), pp. 355-362 (2011).

32. Sotiris, B., Kotsiantis, I., and Zaharakis, P. Pintelas, "Supervised machine learning: A review of classification techniques", Proceedings of the $200^{7}$ Conference on Emerging Artificial Intelligence, pp. 3-24 (2007).

33. Wu, S.-L., Wu, C.-W., Pal, N.R., Chen, C.-Y., Chen, S.-A., and Lin, C.-T. "Common spatial pattern and linear discriminant analysis for motor imagery classification", In Computational Intelligence, Cognitive Algorithms, Mind, and Brain (CCMB), 2013 IEEE Symposium on, IEEE, pp. 146-151 (2013).

34. Pfurtscheller, G., Neuper, C., Schlogl, A., and Lugger, K. "Separability of EEG signals recorded during right and left motor imagery using adaptive autoregressive parameters", IEEE transactions on Rehabilitation Engineering, 6(3), pp. 316-325 (1998).

35. Ince, N.F., Goksu, F., Tewfik, A.H., and Arica, S. "Adapting subject specific motor imagery EEG patterns in spacetimefrequency for a brain computer interface", Biomedical Signal Processing and Control, 4(3), pp. 236-246 (2009).

36. Lal, T.N., Schroder, M., Hinterberger, T., Weston, J., Bogdan, M., Birbaumer, N., and Scholkopf, B. "Support vector channel selection in BCI", IEEE Transactions on Biomedical Engineering, 51(6), pp. 1003-1010 (2004). 
37. Garcia, G.N., Ebrahimi, T., and Vesin, J.-M. "Support vector EEG classification in the Fourier and time-frequency correlation domains", In Neural Engineering, 2003. Conference Proceedings. First International IEEE EMBS Conference on, IEEE, pp. 591-594 (2003).

38. Subasi, A. and Gursoy, M.I. "EEG signal classification using PCA, ICA, LDA and support vector machines", Expert Systems with Applications, 37(12), pp. 86598666 (2010).

39. Bennett, K.P. and Campbell, C. "Support vector machines: hype or hallelujah?", ACM SIGKDD Explorations Newsletter, 2(2), pp. 1-13 (2000).

40. Lotte, F., Congedo, M., Lécuyer, A., Lamarche, F., and Arnaldi, B. "A review of classification algorithms for EEG-based brain-computer interfaces", Journal of Neural Engineering, 4(2), pp. R1-R13 (2007).

41. Nicolas-Alonso, L.F. and Gomez-Gil, J. "Brain computer interfaces, a review", Sensors, 12(2), pp. 12111279 (2012).

42. Bhattacharyya, S., Khasnobish, A., Chatterjee, S., Konar, A., and Tibarewala, D.N. "Performance analysis of LDA, QDA and KNN algorithms in left-right limb movement classification from EEG data", In Systems in Medicine and Biology (ICSMB), 2010 International Conference on, IEEE, pp. 126-131 (2010).

43. Kayikcioglu, T. and Aydemir, O. "A polynomial fitting and k-NN based approach for improving classification of motor imagery BCI data", Pattern Recognition Letters, 31(11), pp. 1207-1215 (2010).

44. Shyu, K.-K., Chiu, Y.-J., Lee, P.-L., Lee, M.-H., Sie, J.-J., Wu, C.-H., Wu, Y.-T., and Tung, P.-C. "Total design of an FPGA-based braincomputer interface control hospital bed nursing system", IEEE Transactions on Industrial Electronics, 60(7), pp. 2731-2739 (2013).

45. Feng, C.-W., Hu, T.-K., Chang, J.-C., and Fang, W.C. "A reliable brain computer interface implemented on an FPGA for a mobile dialing system", 2014 IEEE International Symposium on Circuits and Systems (IS$C A S)$. IEEE (2014).

46. Shyu, K.-K., Lee, P.-L., Lee, M.-H., Lin, M.-H., Lai, R.-J., and Chiu, Y.-J. "Development of a lowcost FPGA-based SSVEP BCI multimedia control system", IEEE Transactions on Biomedical Circuits and Systems, 4(2), pp. 125-132 (2010).

47. Kais, B., Ghaffari, F., Romain, O., and Djemal, R. "An embedded implementation of home devices control system based on brain computer interface", 26th International Conference on Microelectronics (ICM), IEEE (2014).

48. Belwafi, K., Ghaffari, F., Djemal, R., and Romain, O. "A hardware/software prototype of EEG-based BCI system for home device control", Journal of Signal Processing Systems, pp. 1-17 (2016).

49. Liao, L.-D., Wang, I.-J., Chang, C.-J., Lin, B.S., Lin, C.-T., and Tseng, K.C. "Human cognitive application by using wearable mobile brain computer interface", In 2010 IEEE Region 10 Conference TENCON, pp. 346-351 (2010). https://ieeexplore. ieee.org/abstract/document/5686646

50. Shyu, K.-K., Lee, P.-L., Lee, M.-H., Lin, M.-H., Lai, R.-J., and Chiu, Y.-J. "Development of a lowcost FPGA-based SSVEP BCI multimedia control system", IEEE Transactions on Biomedical Circuits and Systems, 4(2), pp. 125-132 (2010).

51. Gao, X., Xu, D., Cheng, M., and Gao, S. "A BCI-based environmental controller for the motiondisabled", IEEE Transactions on Neural Systems and Rehabilitation Engineering, 11(2), pp. 137-140 (2003).

52. Shyu, K.-K., Chiu, Y.-J., Lee, P.-L., Lee, M.-H., Sie, J.-J., Wu, C.-H., Wu, Y.-T., and Tung, P.-C. "Total design of an FPGA-based braincomputer interface control hospital bed nursing system", IEEE Transactions on Industrial Electronics, 60(7), pp. 2731-2739 (2013).

53. BCI Competitions: http://www.bbci.de/competition.

54. http://www.bbci.de/competition/iii/results/

55. http://www.bbci.de/competition/iv/results/

56. Niedermeyer, E. and da Silva, F.L., Electroencephalography: Basic Principles, Clinical Applications, and Related Fields, Lippincot Williams and Wilkins (2004).

57. Yates, R. and Lyons, R. "DC Blocker Algorithms [DSP Tips \& Tricks]", IEEE Signal Processing Magazine, 25(2), pp. 132-134 (2008).

58. Babiloni, F., Cincotti, F., Carducci, F., Rossini, P.M., and Babiloni, C. "Spatial enhancement of EEG data by surface Laplacian estimation: the use of magnetic resonance imaging-based head models", Clin Neurophysiol, 112(5), pp. 724-727 (2001).

59. Murugappan, M., Nagarajan, R., and Yaacob, S. "Combining spatial filtering and wavelet transform for classifying human emotions using EEG Signals", Journal of Medical and Biological Engineering, 31(1), pp. 45-51 (2011).

60. Pfurtscheller, G. and Da Silva, F.H.L "event-related EEG/MEG synchronization and desynchronization: basic principles", Clinical Neurophysiology, 110(11), pp. 1842-1857 (1999).

61. Hashimoto, Y. and Ushiba, J. "EEG-based classification of imaginary left and right foot movements using beta rebound", Clinical Neurophysiology, 124(11), pp. 2153-2160 (2013).

62. Dennis, J., McFarland, Lynn M., McCane, Stephen V,. David, Jonathan R., Wolpaw, "Spatial filter selection for EEG-based communication", Electroencephalography and Clinical Neurophysiology, pp. 386-394 (1997).

63. Tian, L., Erdogmus, D., Adami, A., Pavel, M., and Mathan, S., "Salient EEG channel selection in brain computer interfaces by mutual information maximization", in Proc. IEEE/ EMBS, pp. 7064-7067 (2005).

64. Shadvar, A. "Dimension Reduction by Mutual Information FEATURE EXTRACTION", International Journal of Computer Science \& Information Technology (IJCSIT), 4(3), pp. 13-24 (June 2012). 
65. Balagani, K.S. and Phoha, V.V. "On the feature selection criterion based on an approximation of multidimensional mutual information", in IEEE Transactions on Pattern Analysis and Machine Intelligence, 32(7), pp. 1342-1343 (July 2010).

66. Friedman, J., Hastie, T., and Tibshirani, R., The Elements of Statistical Learning, Data Mining, Inference, and Prediction, Springer, Second Edition (2008).

67. Nicolas-Alonso, L.F. and Gomez-Gil, J. "Brain computer interfaces, a review", Sensors, Molecular Diversity Preservation International, 12(2), pp. 1211-1279 (2012).

68. Burges, C.J.C. "A tutorial on support vector machines for pattern recognition", Data Min. Knowl. Discov., 2, p. 121167 (1998).

69. Allison, B., Graimann, B., and Pfurtscheller, G. "Brain-computer interfaces: Revolutionizing humancomputer interaction", a chapter of Digital Signal Processing and Machine Learning, Springer Science \& Business Media (2010).

\section{Biographies}

Alireza Malekmohammadi received the BSc and MSc degrees in Electrical Engineering from Shahid Beheshti University and Sharif University of technology, Tehran, Iran in 2013 and 2015, respectively. His research interests include machine learning, braincomputer Interface, image processing, and VLSI Implementation of biomedical signal processing algorithms.

Hoda Mohammadzade is an Assistant Professor at the Electrical Engineering Department at the Sharif University of Technology, Tehran, Iran. She received her BSc degree from Amirkabir University of Technology (Tehran Polytechnic), Iran in 2004, the MSc degree from the University of Calgary, Canada in 2007, and the $\mathrm{PhD}$ degree from the University of Toronto, Canada in 2012, all in Electrical Engineering. She is currently an Assistant Professor of Electrical Engineering at Sharif University of Technology, Tehran, Iran. Her research interests include signal and image processing, computer vision, pattern recognition, biometric systems, and bioinformatics.

Alireza Chamanzar received the BSc and MSc degrees in Electrical Engineering, specializing in Electronics and Signal Processing from Sharif University of Technology, Tehran, Iran in 2014 and 2016, respectively. His research interests include algorithm development, system level designs in biomedical applications, and machine learning.

Mahdi Shabany is an Associate Professor in the Electrical Engineering Department at the Sharif University of Technology, Tehran, Iran. He received the BSc degree in Electrical Engineering from Sharif University of Technology, Tehran, Iran in 2002, and his MSc and $\mathrm{PhD}$ degrees both in the same major from the University of Toronto, Toronto, Canada in 2004 and 2008, respectively. He also works at the University of Toronto periodically as a visiting researcher. From 2007 to 2008, he was with Redline Communications Co., Toronto, Canada, where he developed and patented designs for WiMAX systems. He also served as a post-doctoral fellow at the University of Toronto in 2009. He holds three US patents. His main research interests include Digital Electronics, VLSI architecture/algorithm design for broadband communication systems, efficient implementation of signal processing algorithms for various applications including imaging, and bio-oriented systems.

Benyamin Ghojogh obtained his first and second BSc degrees in Electrical Engineering (Electronics and Telecommunications fields) from Amirkabir University of Technology, Tehran, Iran in 2015 and 2017, respectively. He also received his MSc degree in Electrical Engineering (Digital Electronic Systems field) from Sharif University of Technology, Tehran, Iran in 2017. One of his honors is taking the second rank of Electrical Engineering Olympiad of Iran in 2015. His research interests include machine learning and computer vision. 